\title{
Research Paper \\ Prediction of Psychological Well-Being in the Elderly by Assessing their Spirituality, Gratitude to God, and Perceived Social Support
}

\author{
${ }^{*}$ Majid Sadoughi ${ }^{1}$ (1) Fatemeh Hesampour $^{2}$ (B)
}

1. Department of Psychology, Faculty of Humanities, University of Kashan, Kashan, Iran

2. Department of Psychology, Faculty of Psychology \& Educational Sciences, University of Semnan, Semnan, Iran.

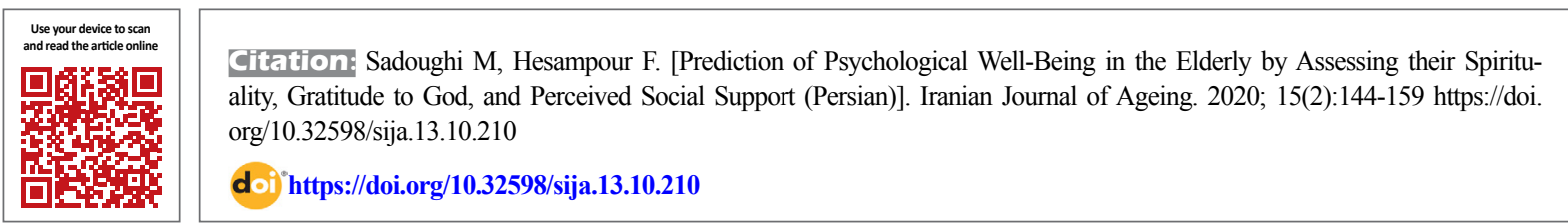

\section{(i) (3)}

Received: 25 Sep 2017 Accepted: 02 Jul 2018 Available Online: 01 July 2020

Key words: Psychological wellbeing, Spirituality, Gratitude to God, Social support, Elderly

\section{A B S T R A C T}

Objectives Today, psychological care for the elderly has received increasing attention due to potentia threats posed by aging, loneliness, gradual decline in physical activity, increase of chronic diseases, social isolation, and physical and mental disabilities. The positive psychology approach to mental health seeks to promote the mental health of people by identifying and developing mental abilities and competences. The present study aimed to predict psychological well-being of the elderly based on the components of positive psychology including spirituality, gratitude to God, and perceived social support.

Methods \& Materials This is a descriptive correlational study conducted on 211 elderly people aged 60-70 years (109 males and 102 females) who were selected randomly. Data collection tools were a demographic form (surveying age, gender, education, marital status, and socioeconomic status), and Paloutzian and Ellisons' Spirituality Well-Being Scale, short form of Ryff's Psychological Wellbeing Scale, Emmons and Crumpler's Gratitude to God Questionnaire, and Zimet's Multidimensional Scale of Perceived Social Support. Collected data were analyzed in SPSS software V. 22 by using descriptive (Mean and Standard Deviation), Pearson correlation test, and stepwise regression analysis.

Results Mean and Standard Deviation of different study variables were reported as follows: Psychological well-being =7.1 \pm 10.68 ; Spirituality =90.22 \pm 15.36 ; Gratitude to God =14.09 \pm 2.41 ; Social support from family members $=21.6 \pm 4.2$; Social support from friends $=16.5 \pm 5.8$; and Social support from significant others $=20.23 \pm 5$. The results of stepwise regression analysis indicated that spirituality $\left(F_{1,209}=62.02, P=0.001\right)$, social support from family members $\left(F_{1,208}=11.06, P=0.001\right)$, and gratitude to $\operatorname{God}\left(F_{1,207}=4.80, P=0.001\right)$ could significantly explain $27 \%$ of the psychological well-being variance in the elderly.

Conclusion Increased spirituality, gratitude to God, and perceived social support especially from family members can improve psychological well-being of the elderly. Hence, Strengthening and paying special attention to their spiritual needs and perceived social support as well as planning health care for them can help increase their psychological well-being.

\section{* Corresponding Author:}

Majid Sadoughi, PhD.

Address: Department of Psychology, Faculty of Humanities, University of Kashan, Kashan, Iran

Tel: +98 (31) 55913750

E-mail: sadoughi@kashanu.ac.ir 


\section{Extended Abstract}

\section{Introduction}

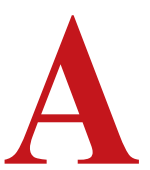

ging is one of the most challenging stages of life; a complicated set of physical, psychological, and social changes impose a heavy burden on the elderly's psycho-social functioning and wellbeing. Previous studies have shown that positive psychological traits such as positive affect, positive relations, and meaning in life have a significant relationship with better psychological well-being outcome. Along with the development of positive psychology in the last decade, a new perspective in the field of geriatrics has been developed which is in contrast with common negative perspective. It is based on new concepts related to aging such as psychological well-being, happiness, life satisfaction, social support, spirituality, and positive aging.

There are various important factors for the well-being and life satisfaction of the elderly, one of which is spiritual and religious coping strategies. Spirituality is defined as the sense of receiving meaning or purpose from a higher source and is related to a person's perception of health and well-being. Spirituality has a key role in coping with stress and mental distress. In fact, religious beliefs, especially when combined with group activities, can prevent loneliness and reduce depression, anxiety, and other psychological symptoms.

Gratitude to God is another component of positive psychology that is expected to be associated with the psychological well-being of the elderly. Studies have shown that gratitude is one of the most beneficial positive personality traits. McAdams et al. [22] believe that the gratitude is more importance for the elderly, since it helps them better pass through their last stages of adulthood and achieve cohesion. On the other hand, perceived social support has a relationship with the elderly's psychological well-being. In fact, social support is one of the most important forms of social relations and can be different in individuals considering their age, gender, personality, and even culture. At each stage of life, some aspects of social support may become more important and more effective.

There is scanty empirical evidence on positive aging in Iranian samples and there are few studies have explored the effect of positive psychology components on psychological well-being of the elderly. Therefore, the present study is an attempt to examine the relationship of psy- chological well-being with spirituality, gratitude to God, and perceived social support in the elderly, also measure the contribution of each of these variables in predicting psychological well-being of the elderly.

\section{Methods \& Materials}

This is a descriptive-correlational study. Study population consists of all older people aged $60-75$ years in Kashan, Iran in 2016. Of these, 211 participants were participated in the study and filled out Paloutzian and Ellisons' Spirituality Well-Being Scale (SWBS), short form of Ryff's Psychological Wellbeing Scale (PWBS), Emmons and Crumpler's Gratitude to God Questionnaire (GGQ), and Zimet's Multidimensional Scale of Perceived Social Support (MSPSS) . In addition, a demographic form was used to record their information including age, marital status, education, and socio-economic status.

The SWBS has 20 items rated on a 6-point Likert scale ranging from 'completely disagree' to 'completely agree'. In our study, the Cronbach's alpha coefficient for the internal consistency of its two sub-scales of religious wellbeing and existential well-being were obtained 0.84 and 0.73 , respectively. The GGQ has 4 items rated on a 5-point Likert scale with a Cronbach's alpha of 0.96 reported by Emmons' et al. [19]. In our study, it was obtained 0.76, indicating the high reliability of its Persian version. The MSPSS measures perceived social support received from family members, friénds, significant others. It includes 12 phrases, and its good validity and reliability have been reported in other studies. In the current study, the Cronbach's alpha coefficients for its three sub-scales of support from significant others, family members, and friends were obtained $0.70,0.72$, and 0.83 , respectively. Finally, the short form of PWBS has 18 items rated on a 6-point Likert scale. The Cronbach's alpha for this scale was obtained 0.71 in the present study, showing its satisfactory reliability.

\section{Results}

Mean \pm SD for different study variables and their components are as follows: Psychological well-being $=7.1 \pm 10.68$; Spirituality $=90.22 \pm 15.36$; Gratitude to $\operatorname{God}=14.09 \pm 2.41$; Social support from family members $=21.6 \pm 4.2$; Social support from friends $=16.5 \pm 5.8$; and Social support from significant others $=20.23 \pm 5$. The results of stepwise regression analysis indicated that spirituality $\left(\beta=0.47, \Delta \mathrm{R}^{2}=0.22\right.$, $\mathrm{F}_{1,209}=62.02, \mathrm{P}=0.001$ ), social support from family members $\left(\beta=0.21, \Delta \mathrm{R}^{2}=0.039, \mathrm{~F}_{1,208}=11.06, \mathrm{P}=0.001\right)$ and gratitude to $\operatorname{God}\left(\beta=0.16, \Delta \mathrm{R}^{2}=0.017, \mathrm{~F}_{1,207}=4.80, \mathrm{P}=0.001\right)$ could significantly explain $27 \%$ of the psychological well-being variance in the elderly. 


\section{Conclusion}

The results of the present study are in line with those of Garsen [16], Wilt [38], and Moon \& Kim [39] who reported a direct relationship between spirituality and psychological well-being in the elderly. Spirituality is known as the indicator of significant positive outcome of well-being and quality of life. Spirituality can be very useful as a positive strategy for increasing meaning in life, hope, and social interactions and coping with psychological problems such as depression and loneliness in the elderly. Cowlishaw et al. [41] found that spirituality has a significant relationship of life satisfaction with psychological well-being of the elderly, leading to their better understanding and positive appraisal of life events.

The findings of this study indicated a positive relationship between gratitude to God and psychological well-being, which is consistent with the results of Booker et al. [46], Ramírez [47], Sapmaz et al. [48]. Krause [49] showed that gratitude to God helps the elderly to effectively deal with undesirable life events. A study suggested that gratitude is an important force that should be strengthened in the elderly because it may help them experience less fear and anxiety about death by making them realize they may have a good life [51]. Other studies on different samples of the elderly have shown that those who express gratitude can better prioritize positive aspects of life [18]. These people spend more time thinking about the positive aspects of life and the benefits of social interactions [57].

The results of the present study showed a significant relationship between perceived social support received from family members and psychological well-being in the elderly. These results are consistent with those of Sun et al. [55], Lee et al. [26], and Kishimoto et al. [56]. Sun et al. [55] found that relatives and non-relatives play different roles in the lives of the elderly. Family members always play the most supportive role in the psychological well-being of the elderly, while friends usually provide less of such support. Emotional support is provided by both family members and friends. The older people's friends accompany them in social activities more than their children or relatives. Family members, compared to friends, exert more social control and the elderly expect social support from them when needed.

Spiritual assessments, due to cultural differences, are necessary for the centers where health care services for the elderly are designed and provided. In addition to assessing the mental health states of the elderly, improvement of their social support can play an important role in increasing their satisfaction and, thus, their psychological well-being. In overall, it seems that, by strengthening spirituality, gratitude to God, and perceived social support, we can help the elderly to develop strategies to maintain their psychological and physical well-being.

\section{Ethical Considerations}

\section{Compliance with ethical guidelines}

All ethical principles were considered in this article. The participants were informed about the purpose of the research and its implementation stages; they were also assured about the confidentiality of their information; Moreover, They were allowed to leave the study whenever they wish, and if desired, the results of the research would be available to them.

\section{Funding}

This research did not receive any specific grant from funding agencies in the public, commercial, or not-forprofit sectors.

\section{Authors' contributions}

Conceptualization: Fatemeh Hesampour, Majid Sadoughi; Methodology, analysis, research and review: Majid Sadoughi; Writing Manuscript: Fatemeh Hesampour; Supervi-sion: Majid Sadoughi.

\section{Conflicts of interest}

The authors declared no conflicts of interest. 


\title{
يُشيشينى بهزيستى روانشناختى در سالمندان بر اساس معنويت، قدردانى از خدا و حمايت اجتماعى ادراكشده
}

(10) 'مجيد صدوقى' 'D، فاطمه حساميور"

\author{
1. كروه روانشناسى، دانشكده علوم انساني، دانشكاه كاشان، كاشان، ايران.

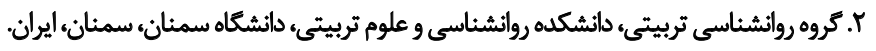

\begin{abstract}
حكSد

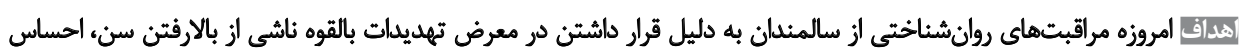

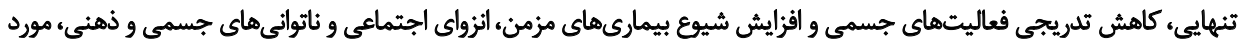

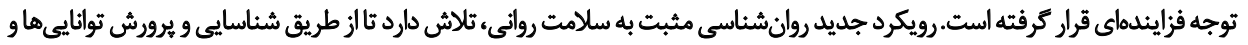

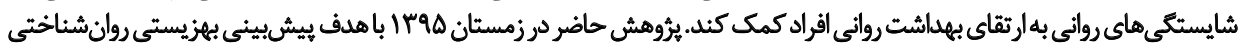

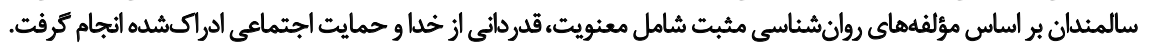

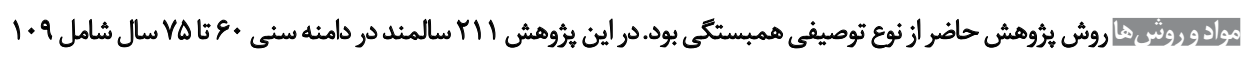

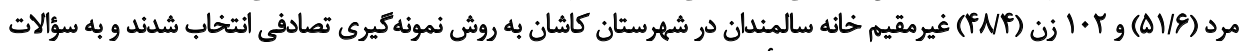

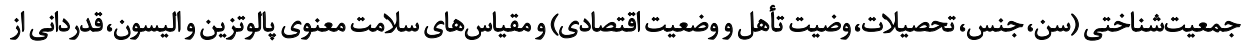

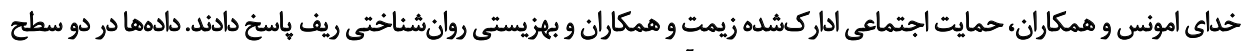

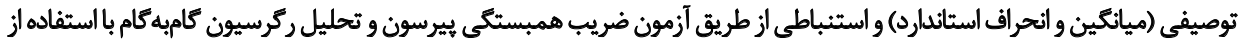

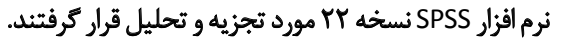

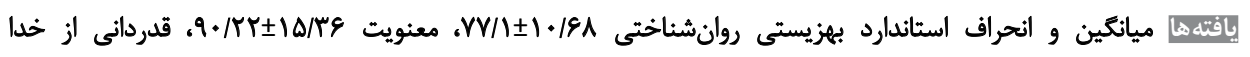

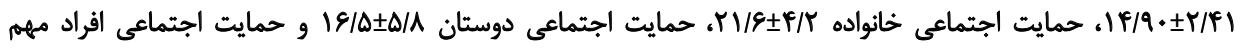
$\Delta R^{2}=$ A و

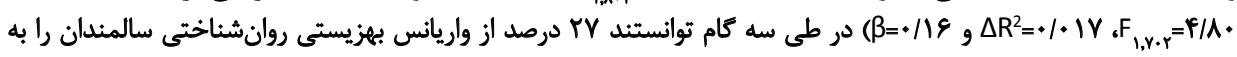
طور معنادارى تبيين كنئد.

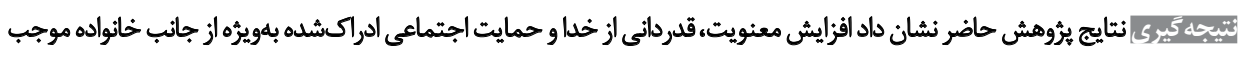

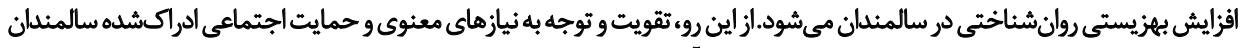

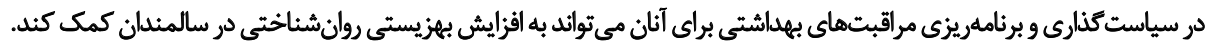

تاريخ دريافت: 1·خرداد وهوبا

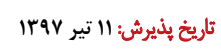

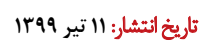

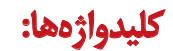

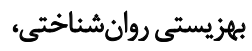
معنويت، قدرداني از روان حدا و حمايت اجتماعيء سالمند

عملكرد جسمانى [T] و بيمارىهاى مزمن روبهرو مىشوند كه

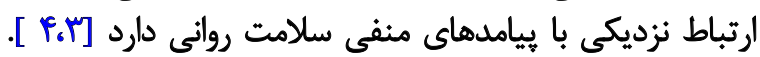

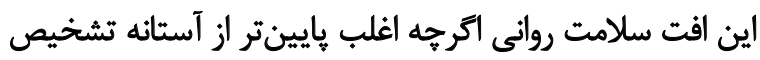

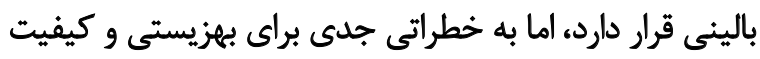

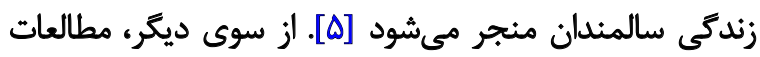

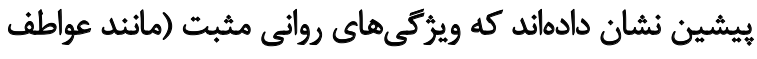

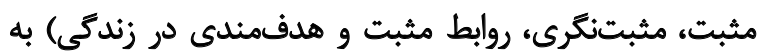

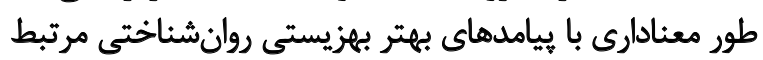

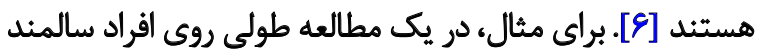

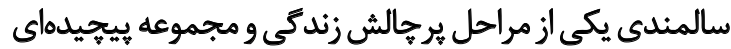

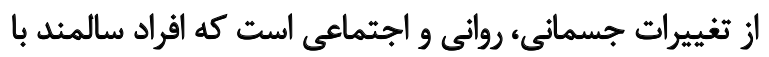

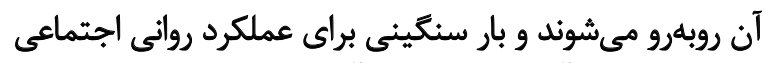

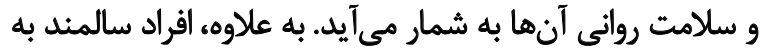

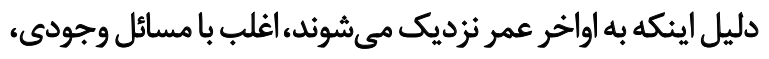

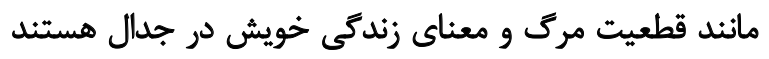

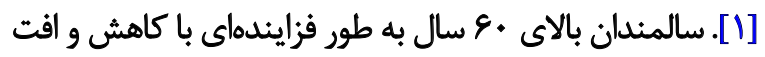


كنار آمدن با استرس و يريشانىهاي روانى سالمندان دارد و بهره

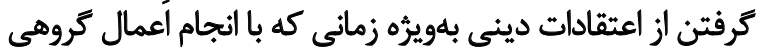

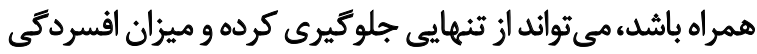

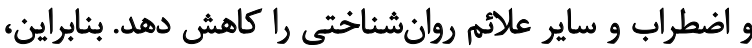

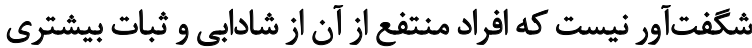

در زندكى برخوردار باشند [19].

يكى ديكر از مؤلفهاهى روانشناسى مثبت كه انتظار ميرود با

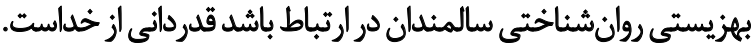

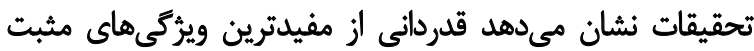

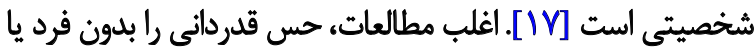

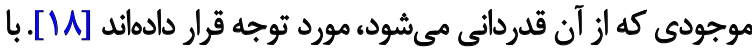

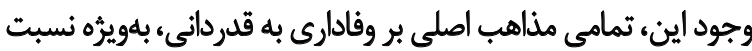

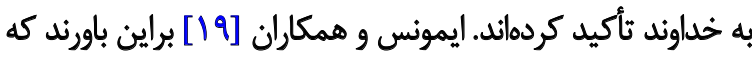

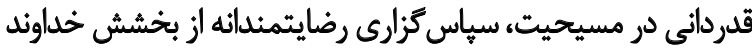

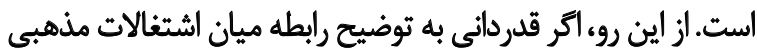

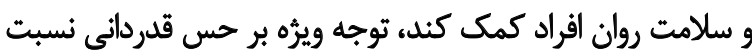

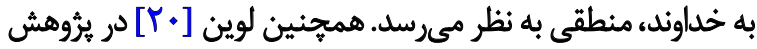

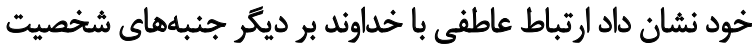

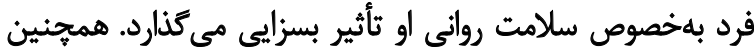

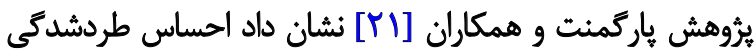

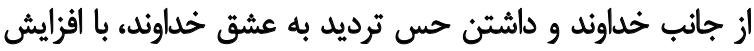

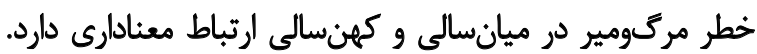

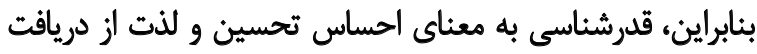

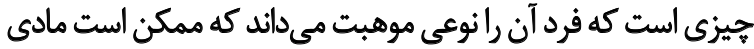

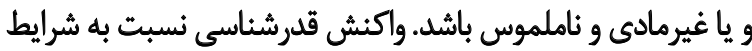

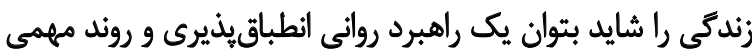

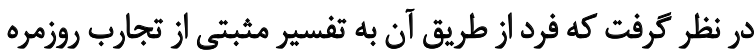

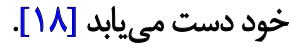

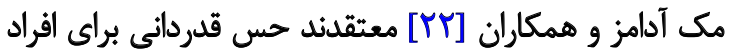

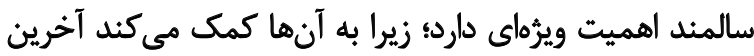

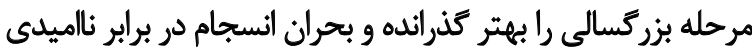

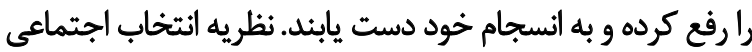

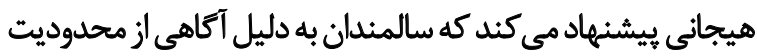

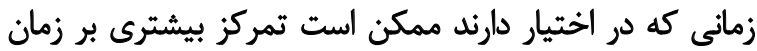

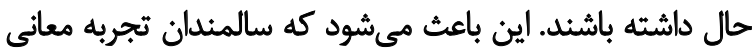

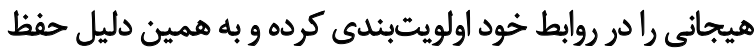

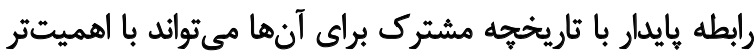

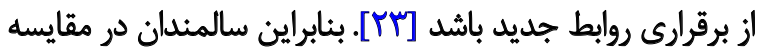

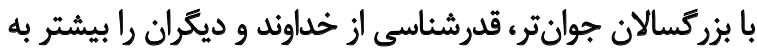

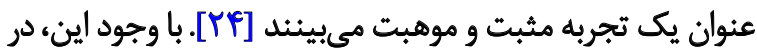

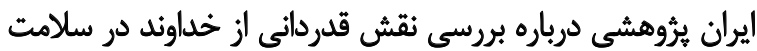

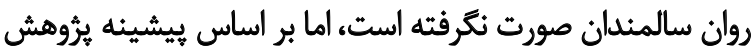

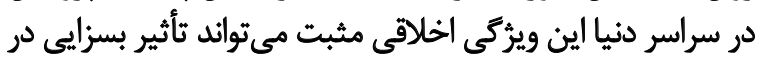
بهزيستى روانشناختى اين قشر از افراد جامعه ايفا كند.

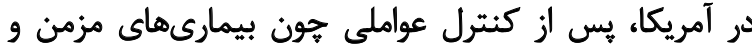

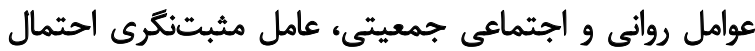

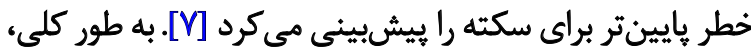

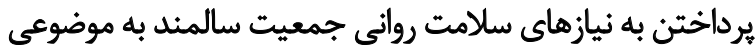

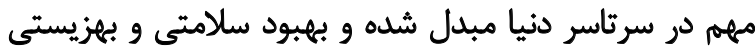

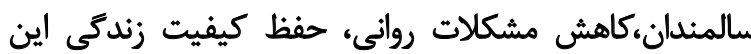

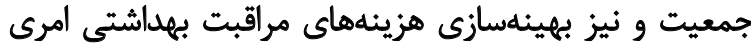

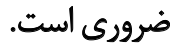

همراستا با توسعه روانشناسى مثبت در دها اخير، ديدكاه

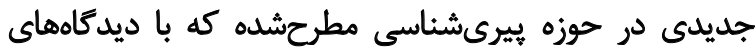

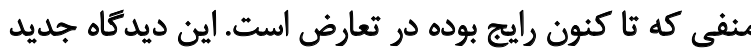

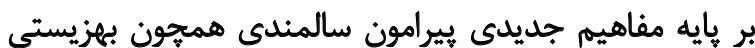

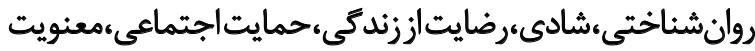

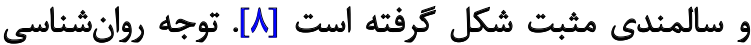

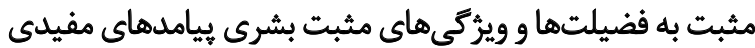

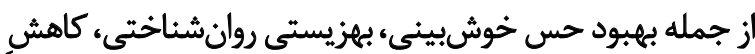

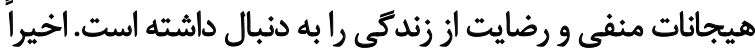

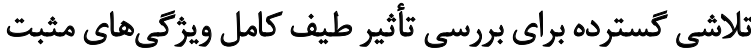

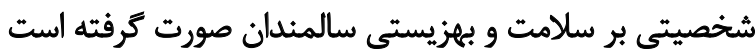

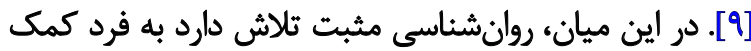

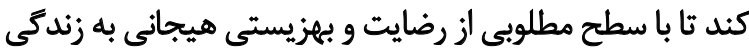

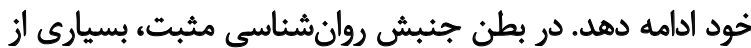

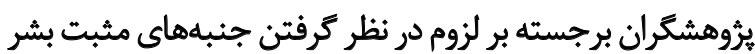
و مفهوم بهزيستى در تعريف سلامت روان تأكيد كردماند [ـ ـ1]. سالمندان آسيب يذيرى بيشترى در برابر از دست دادن شادى

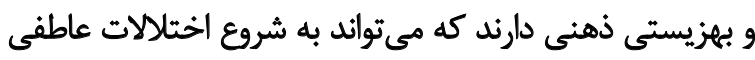

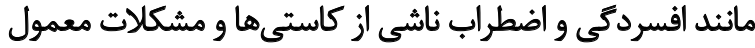

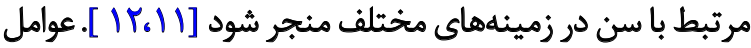

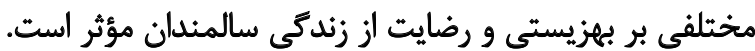

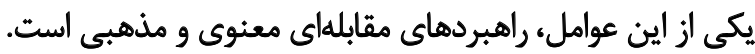

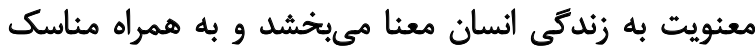

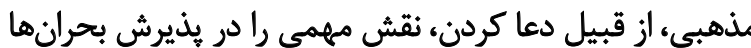

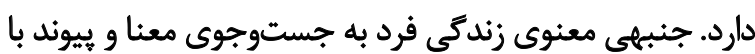

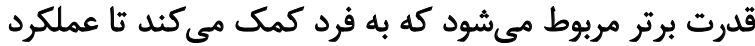

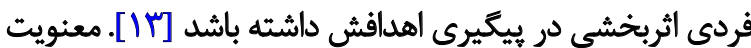

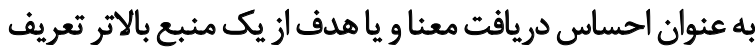

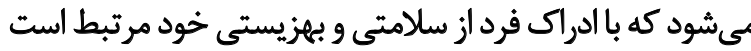

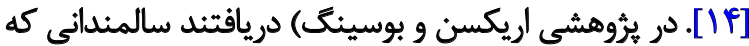

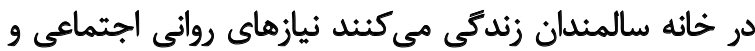

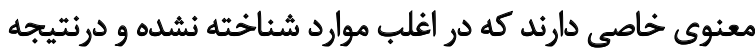

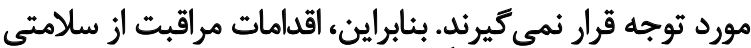

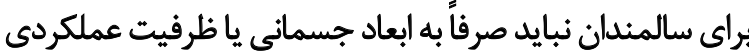

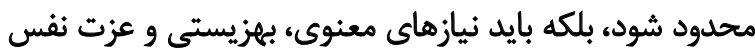

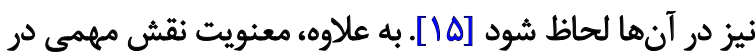


همجون معنويت، قدردانى از خدا و حمايت اجتماعى ادراكشده

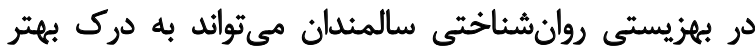

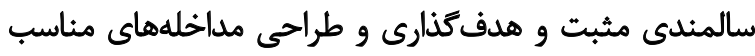

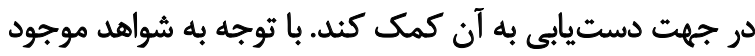

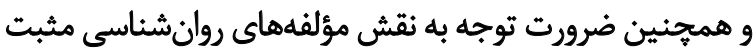

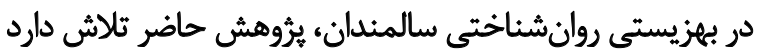

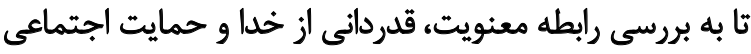

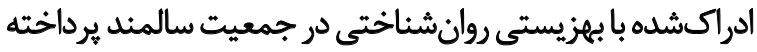

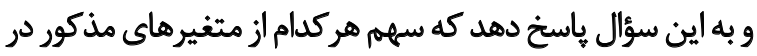

بيشبينى بهزيستى روانشناختى سالمندان جقدر است استى روش مطالعه

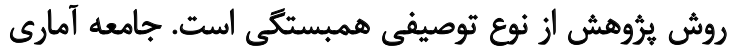

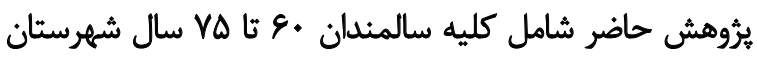

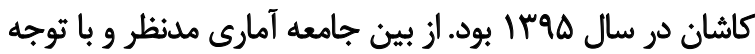

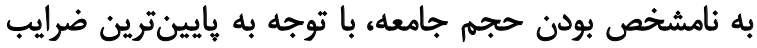

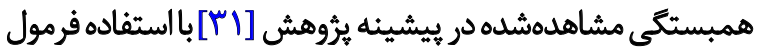

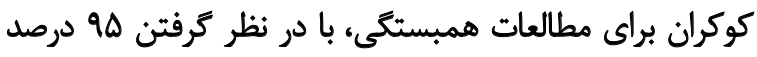

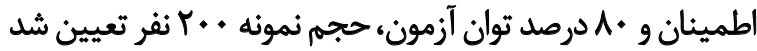

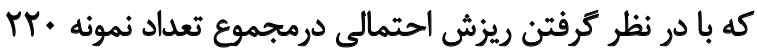

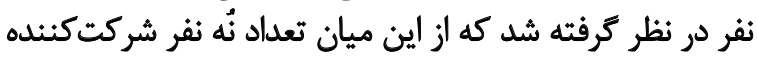

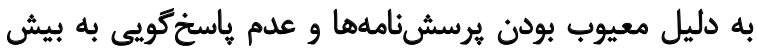

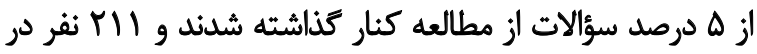

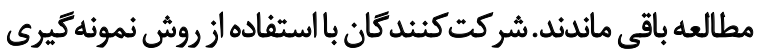

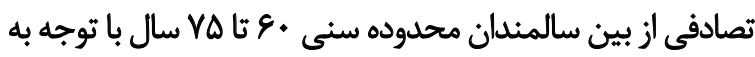
معيارهاى ورود وخروج أنتخاب شدند.

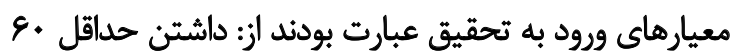

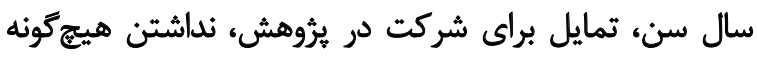

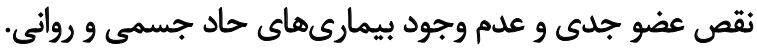

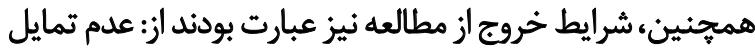

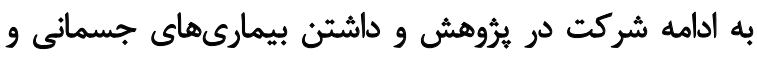

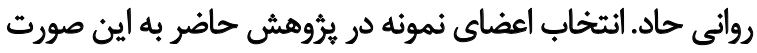

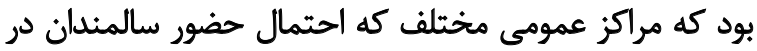

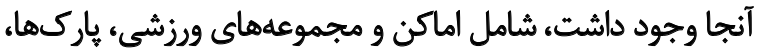

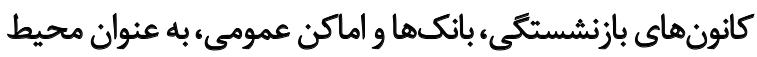

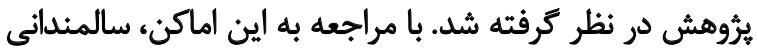

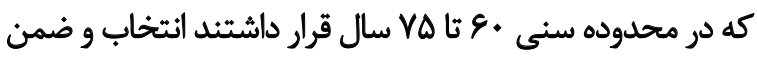

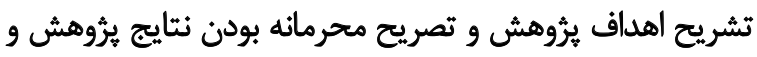

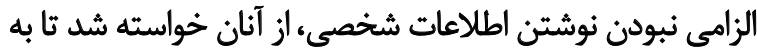

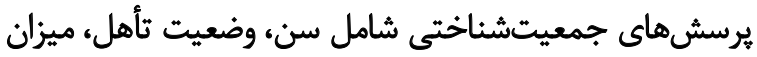

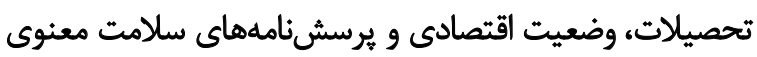

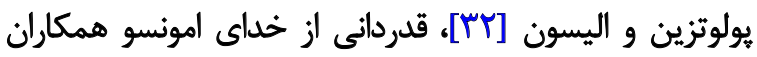

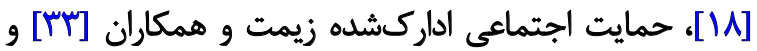

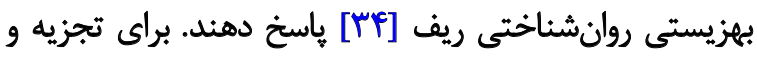

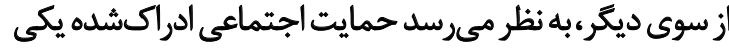

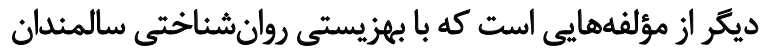

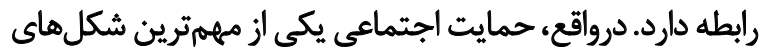

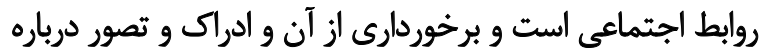

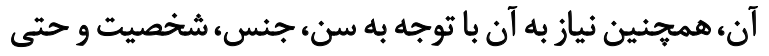

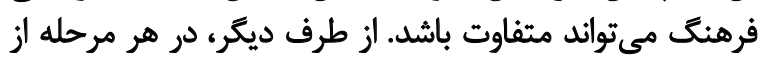

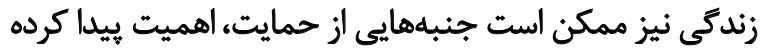

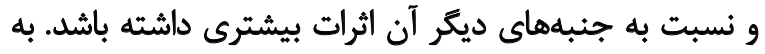

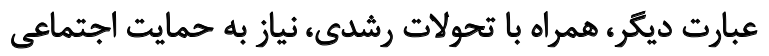

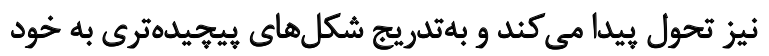

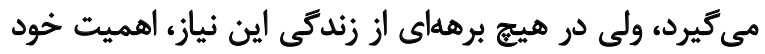

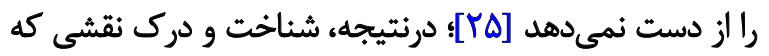

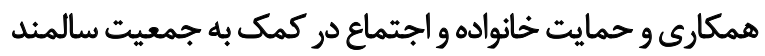

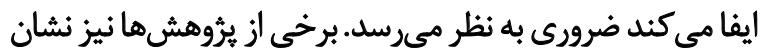

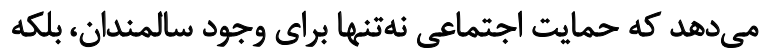

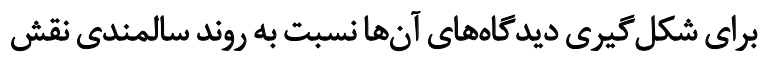

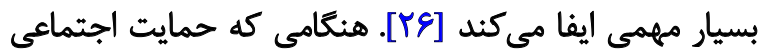

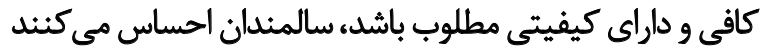

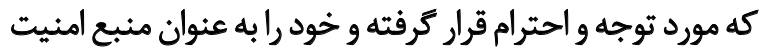

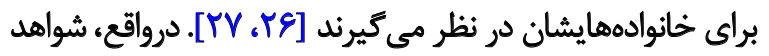

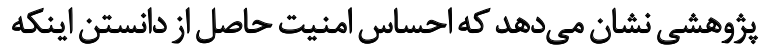

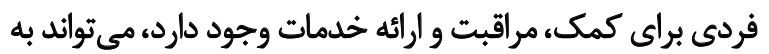

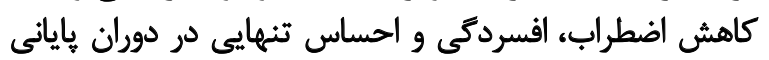

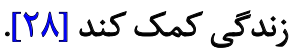

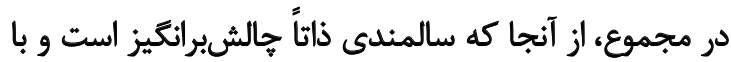

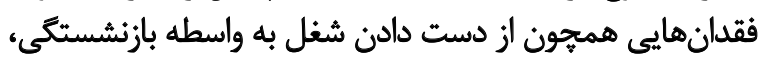

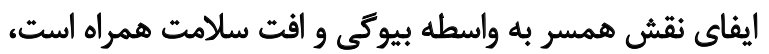

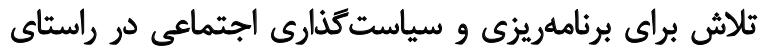

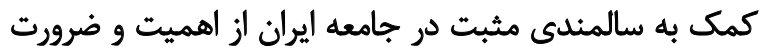

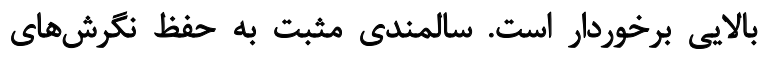

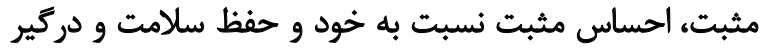

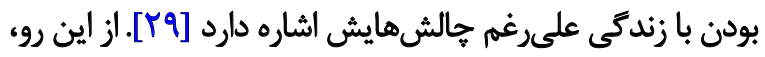

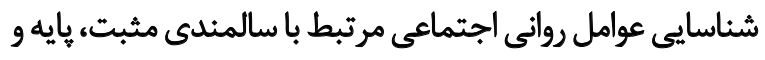

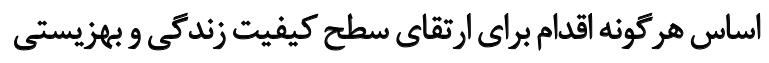

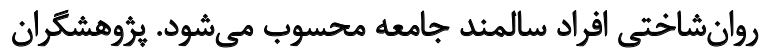

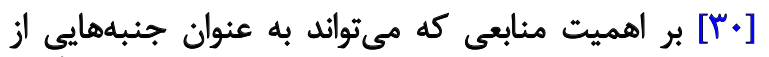

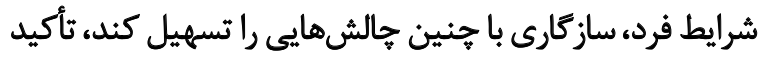

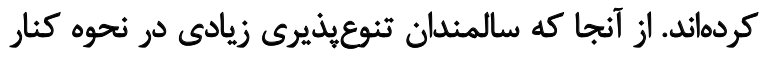

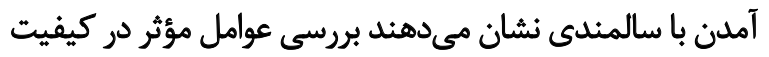

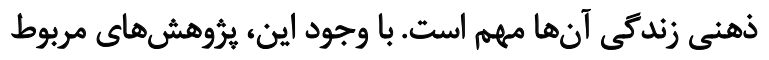

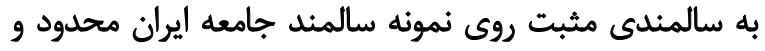

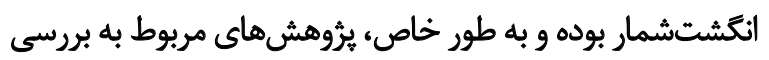

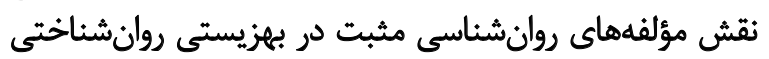

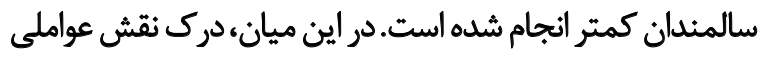


عامل كلى در يرسشنامه قدرداني از خدا بود كه توضيحدهنده

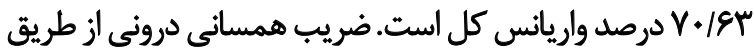

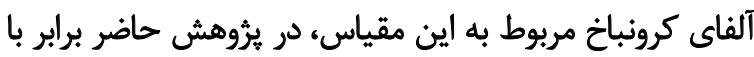

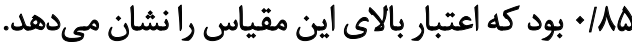

\section{بر سشئامه حمايت إجتماعي ادواكشيده}

يرسشنامه حمايت اجتماعى ادراكشده توسط زيمت و

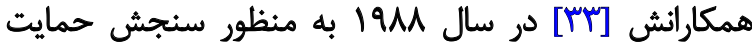

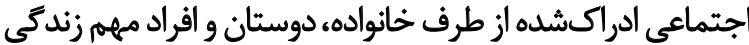

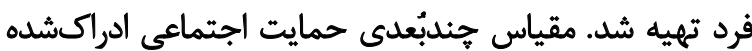

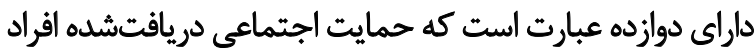

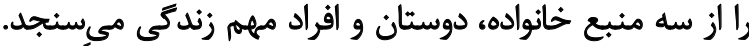

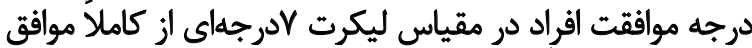

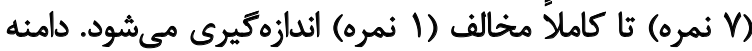

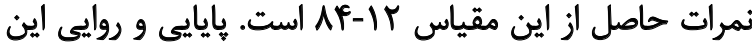

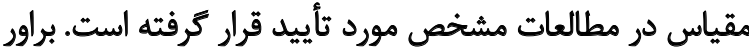

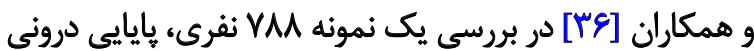

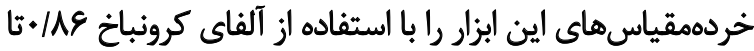

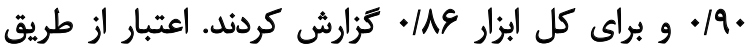

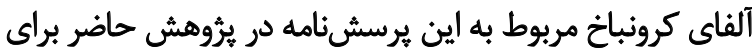

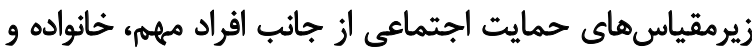

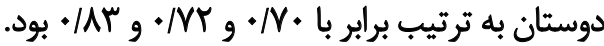

$$
\text { برسش نامه بهزئيستى ووان شُناختى ريف }
$$

نسخه كوتاه هجدهؤالى مقياس بهزيستى روانشناختى ريف

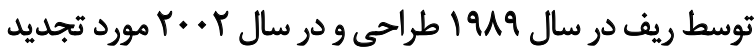

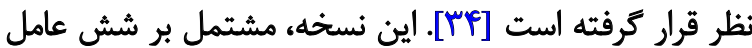

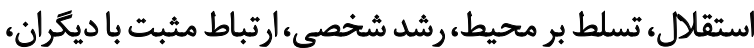

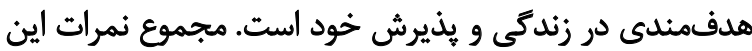

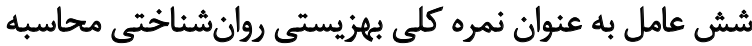

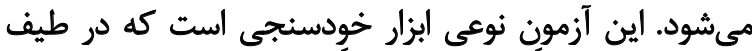

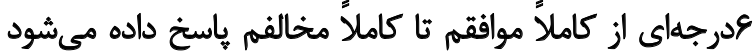

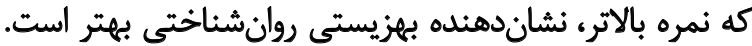

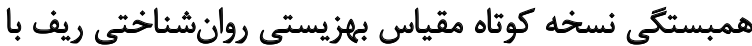

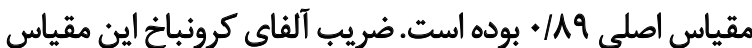

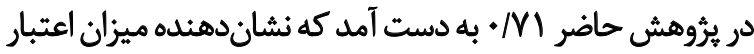
مطلوب اين مقياس است.

ياثتهها

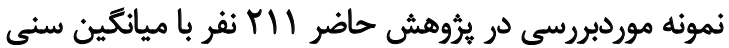
F

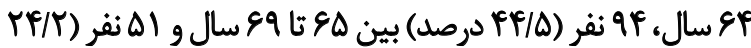

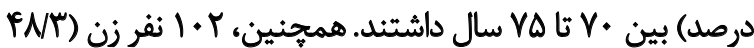

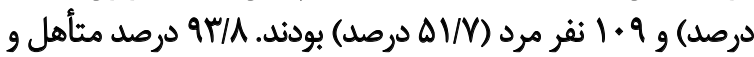

تحليل دادهها در بخش آمار توصيفى از شاخصهاي ميانكين،

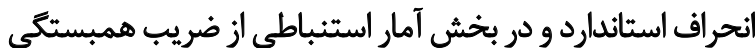

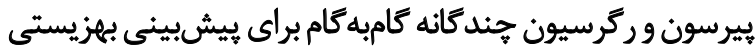

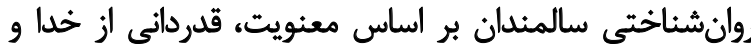

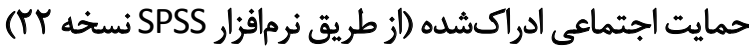

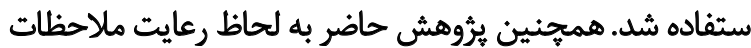

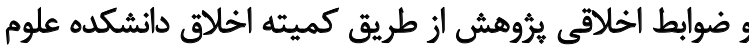
انسانى دانشكاه كاشان مورد بررسى و وتأييد قرار كرفت.

يرسش ئامه سلامث معنوى

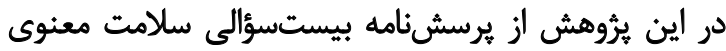

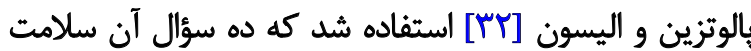

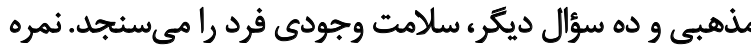

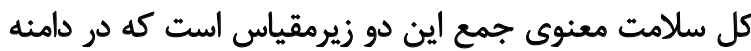

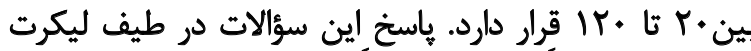

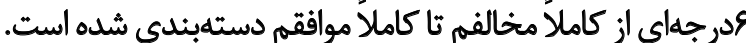

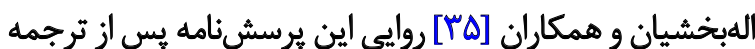

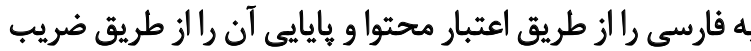

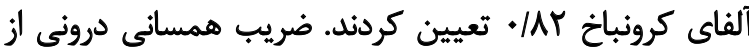

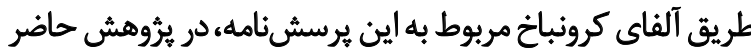

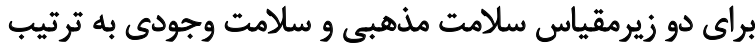

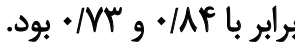

$$
\text { برسش ثامه قدرداني ازخ خداوند }
$$

به منظور سنجش سازه قدردانى از خداوند، از مقياس قدردانى إنى

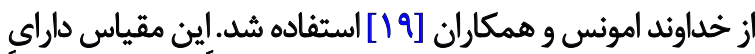

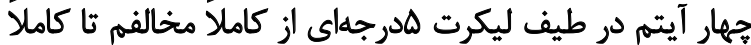

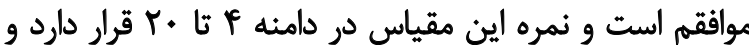

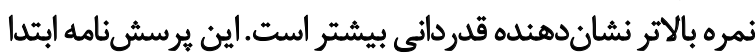

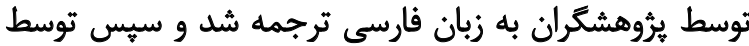

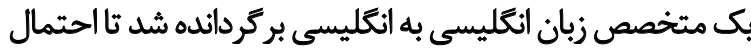

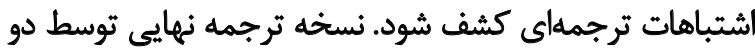

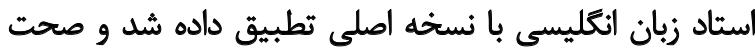

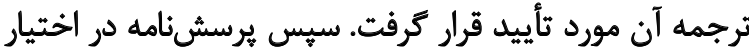

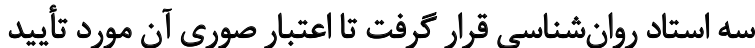

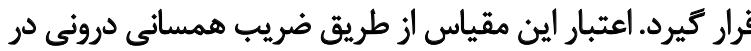

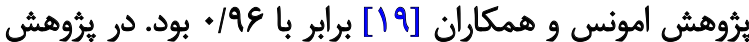

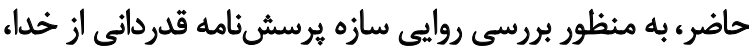

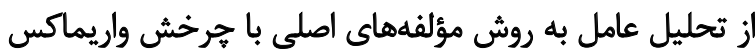

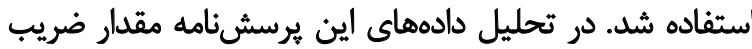

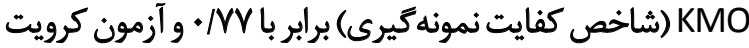

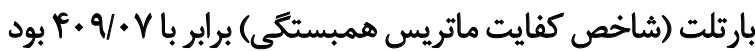

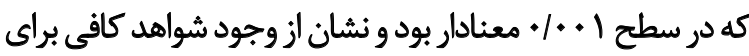

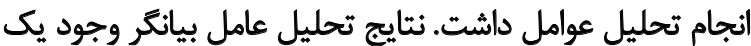


شاخصهاي توصيفى معنويت، قدردانى از خدا و و حمايت

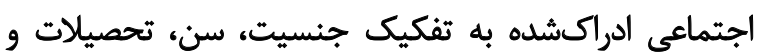
وضعيت تأهل در جدول شماره باده بارئه شده استيت

بر اساس جدول شماره ז، بين مردان و زنان سالمند مورد

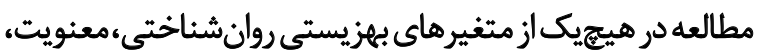

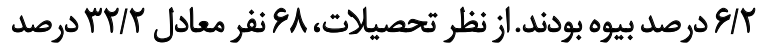

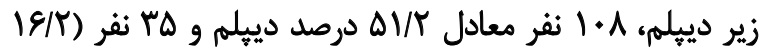

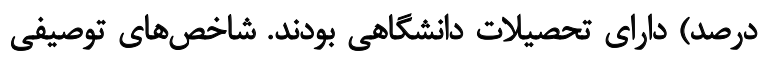

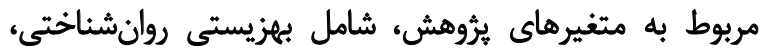

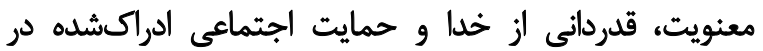
سالمندان موردمطالعه در جدول شماره الران ارائه شده است.

جدول ا. شاخصهاى توصيفى مربوط به بهزيستى روانشناختى، معنويت، قدردانى از خدا و حمايت اجتماعى ادراكشده در سالمندان مورد مطالعه

\begin{tabular}{|c|c|c|c|}
\hline ميانغينين |انحراف استاندارد & ماكزيمه & مينيمه & هتغيرها \\
\hline$W / \pm 1+181$ & 1.1 & $\Delta \Delta$ & بهزيستى روانشناختى \\
\hline$Q . / r Y \pm 10 / r q$ & ilr & $\mathrm{m}$ & معنويت \\
\hline$|\varphi / \cdot q \pm r| r \mid$ & 18 & $p$ & 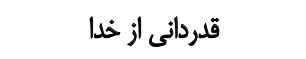 \\
\hline$r V / E \pm F / r$ & ru & $\wedge$ & حمايت اجتماعى خانواده \\
\hline $\mid \varepsilon / \Delta \pm \Delta / \Lambda$ & ru & r & حمايت اجتماعى دوستان \\
\hline$r \cdot / r m \pm \Delta$ & ra & f & حمايت اجتماعى افراد مهم \\
\hline QWPF $\pm M / T \wedge$ & r & r. & حمايت اجتماعى كل ادراكشده \\
\hline
\end{tabular}

L

جدول r. شاخصهاى توصيفى بهزيستى روانشناختى، معنويت، قدردانى ازخدا و حمايت اجتماعى ادراكشده به تفكيك جنسيت، وضعيت تأهل، سن و تحصيلات

\begin{tabular}{|c|c|c|c|c|c|}
\hline \multicolumn{4}{|c|}{ ميانغين土 انحراف استاندارد } & \multirow{2}{*}{\multicolumn{2}{|c|}{ متغير }} \\
\hline حمايت إجتماعى & قدرةاني از مَدا & معنويت & بهزيستى روانشناختي & & \\
\hline$\Delta Y / \Delta \& \pm I / / A$ & $\mid r / \Lambda \cdot \pm r / g 8$ & $\wedge Q / r| \pm| V / q$. & $W / \cdot Y \pm I V / T \Delta$ & مرد & \multirow{2}{*}{ جنسيت } \\
\hline$\Delta 9 / 47 \pm 1 \cdot / 199$ & $|f / r| \pm r / \cdot \Lambda$ & $91 / 4+ \pm \mid r / 11$ & $W / \backslash A Y \pm 9 / q Y$ & ij & \\
\hline $\mathrm{t}=\mathrm{V} / \Lambda \Delta, \mathrm{P}=+/ \mathrm{r \Delta}$ & $\mathrm{t}=\mathrm{V} / \mathrm{Ar}, \mathrm{P}=.1 . \mathrm{gr}$ & $t=-+/ 19, P=+/ 91 \%$ & $\mathrm{t}=-+11+, \mathrm{P}=+/ 91 \mathrm{f}$ & \multicolumn{2}{|r|}{ 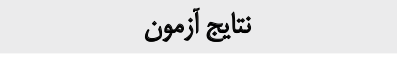 } \\
\hline$\Delta q / \mu T \pm 1 \cdot / \Delta T$ & $\mid f / r \Delta \pm Y / \bullet \varphi$ & $9 r / \circ \& \pm I r / M$ & $W / r v \pm 1+1+\varepsilon$ & متأهل & \multirow{2}{*}{ 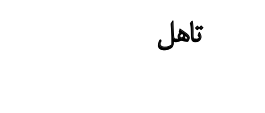 } \\
\hline$M T / \Psi+ \pm I r / \mu f$ & $1 \cdot / M+M / N$ & GT/RHITNIT & $V / q Y \pm \mid g / N$ & 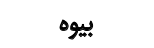 & \\
\hline$t=\Delta / T \Delta, P=.1 . \cdot 1$ & $t=r / \Lambda, P=\bullet / \cdot \cdot r$ & $t=r / A \cdot, P=\cdot / \cdot \cdot r$ & $t=. / q F^{F}, P=\cdot / R g F$ & \multicolumn{2}{|r|}{ نتايج آزمون } \\
\hline$\Delta V / T / \pm 1 \cdot / 91$ & $I r / 9 H \pm Y / Y$. & WEYIIYN. & $V \& / \Delta \pm 1 \cdot 1 \cdot A$ & . & \multirow{3}{*}{ سن } \\
\hline$\Delta Q / N \pm 1.101$ & $\| F / F \cdot \pm T / 10$ & 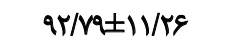 & 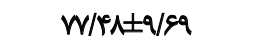 & Pe & \\
\hline$\Delta V / \Delta \cdot \pm I Y / P A$ & $\mid r / M \pm \pm / . r$ & AV/DFETr/Tq & $W / I V \pm I r / A Y$ & سال VA V. & \\
\hline $\mathrm{F}=1 / \cdot \varnothing, \mathrm{P}=. / \mathrm{m}$ & 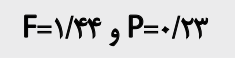 & $F=f / r \cdot, P=* / \cdot I V$ & $\mathrm{~F}=\mathrm{J} / \mathrm{eq} \mathrm{P}=$ =/Af & \multicolumn{2}{|r|}{ نثايج آزمون } \\
\hline QV/YMAIYT/. & leffretr/T. & $M W W \pm 1 \& M^{\circ}$ & $V \Delta / R+ \pm I / / P$ & زير دييلم & \multirow{3}{*}{ تحصيلات } \\
\hline QNATIIV/NQ & $\mid F / \cdot P \pm$ r/aP & $q r / \% q \pm 10 / \cdot V$ & $V N / P \pm 1 . / 19$ & دييلم & \\
\hline$\Delta Q / T A \pm N T \Delta$ & $\mid r / \varepsilon \cdot \pm Y / .$. & $N E / M T \pm I Y / \Delta T$ & $v e / N \pm 1 \cdot / M^{e}$ & دانشكاهي & \\
\hline$F=* / f \Delta_{,} P=* / q T V$ & $\mathrm{~F}=1 / F, \mathrm{P}=\cdot / \pi m \varphi$ & $F=r / \Delta r, P=* / \cdot A r$ & $F=1 / 19, P=* / \% \cdot 9$ & \multicolumn{2}{|r|}{ نثايج آزمون } \\
\hline $11 / 1 r \pm 1 / 89$ & $\|F /\| \pm Y M$. & $\Lambda V M A \pm I F / M T$ & VqeT $\pm 11 / 1 \mathrm{~A}$ & حُوب & \multirow{3}{*}{ وضعيت اقتصادى } \\
\hline $1.1 . \Delta \pm . / A \gamma$ & $|E / r \Delta \pm Y| \cdot \Lambda$ & $97 / .8 \pm \mid f / m$ & $V N \cdot . \pm Q / F V$ & هتوسط & \\
\hline$I r / \Delta A \pm I / R$ & $\mid r / A Y \pm Y / \cdot V$ & AV/TrTIV/YA & $A K / A Y \pm I I / \varphi^{\circ}$ & ضعيف & \\
\hline $\mathrm{F}=. / P \& \mathrm{P}=.18 \mathrm{rr}$ & $F=. / A r P=\cdot / \Delta r g$ & $F=1 / \wedge q, P=\star / \Delta \varphi r$ & $F=F / N, P=* / \bullet 1$ & \multicolumn{2}{|r|}{ نثايج آزمون } \\
\hline
\end{tabular}


جدول ب. ماتريس ضرايب همبستكى بهزيستى روانشئاختى با معنويت، قدردانى از خدا و مؤلفهماى حمايت اجتماعى در سالمندان

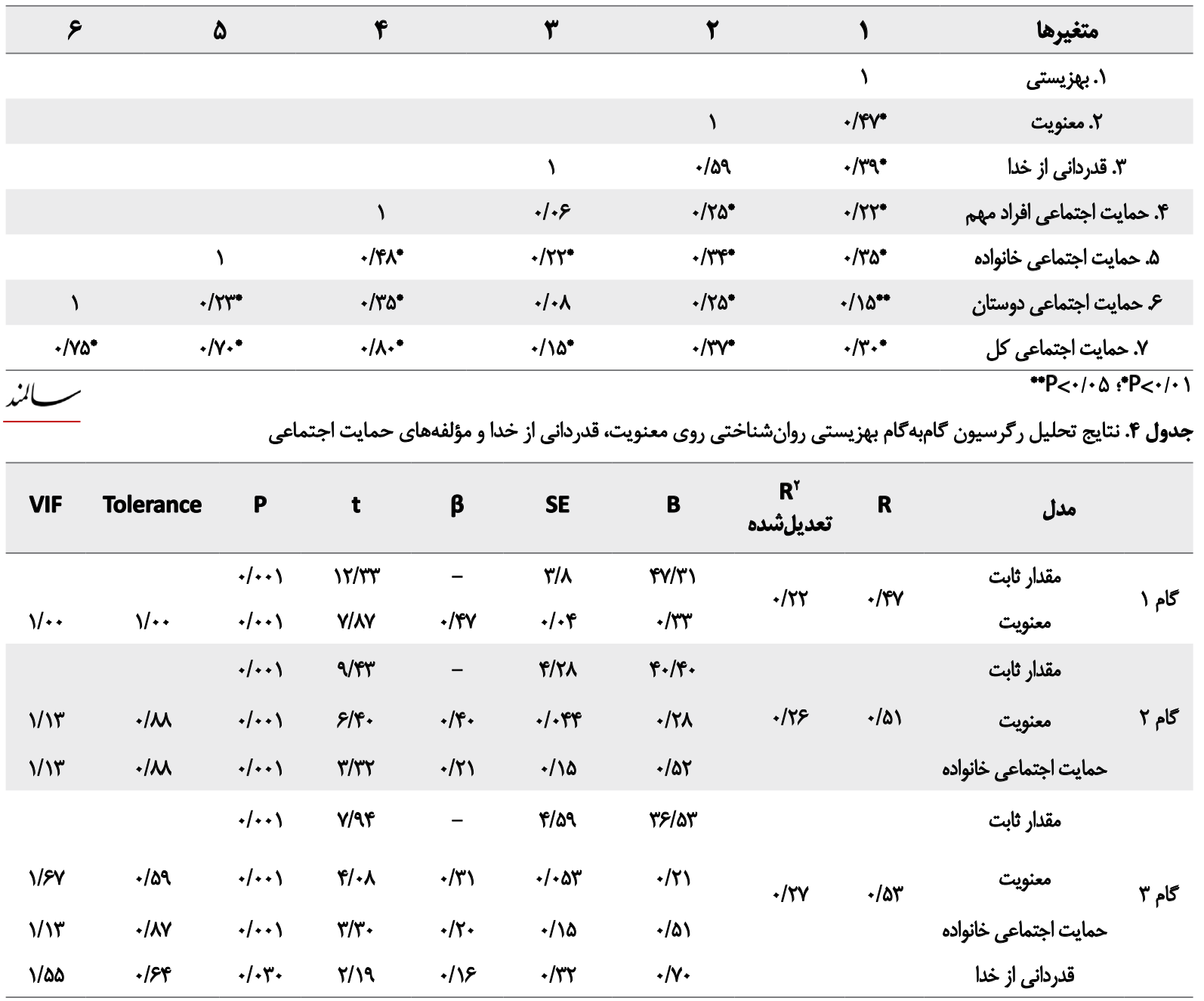

اقتصادى خوب، به طور معنادارى بهزيستي روان شئاختي بالاترى

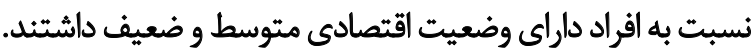

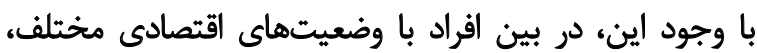

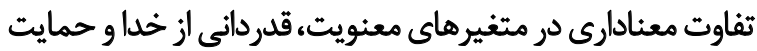

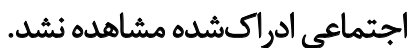

به منظور تعيين رابطه معنويت، قدرداني از خدا و مؤلفههاي

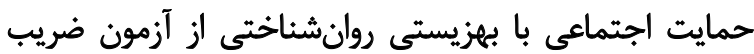

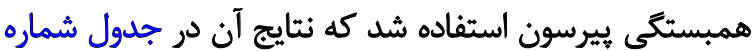
ب آمله اسيث.

همان گونه كه در جدول شماره ب مشاهده ميشود معنويت، مؤلفههاي حمايت اجتماعي خانواده، حمايت اجتماعي دوني دوستان،

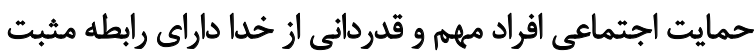

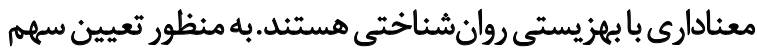

قدرداني از خدا و حمايت اجتماعي ادراكشده ثفاوت معنادارى

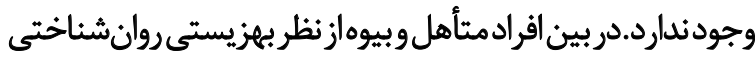

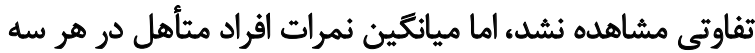

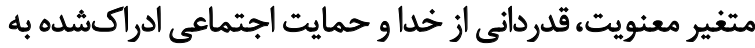

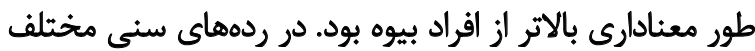

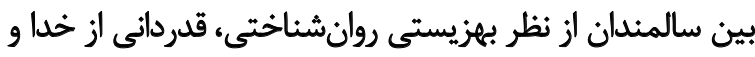

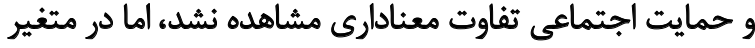

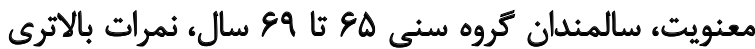
داشتند.در بين افراد با سطوح تحصيلى متفاوت، تفاوت معنادون سناري

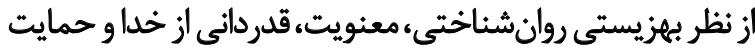
اجتماعى ادراكشده وجود نداشت. به علاوه، تفاوت معنادارى در درئي

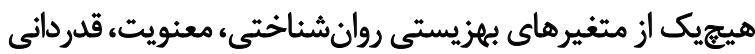

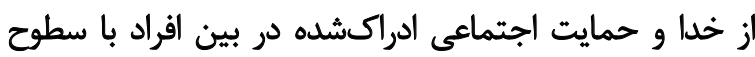

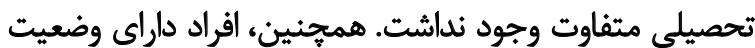




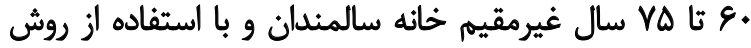

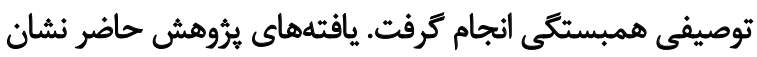

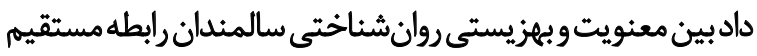

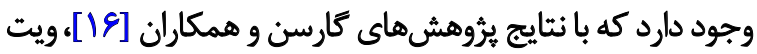

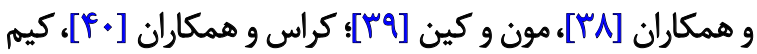

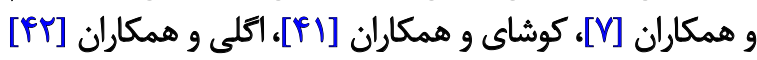

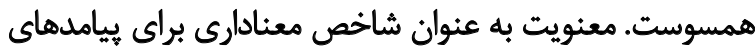

مثبت سلامتى و كيفيت زندكى شناخته شده است [FT]

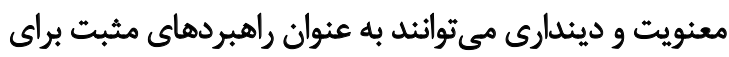

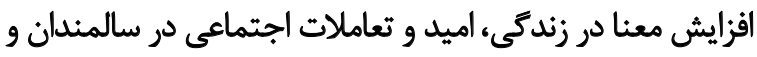

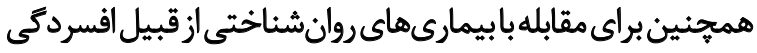

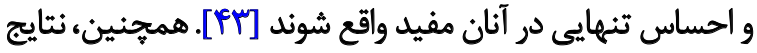

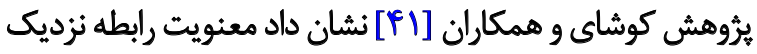

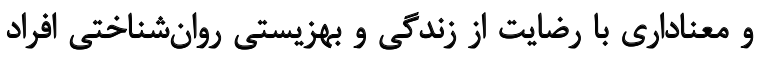

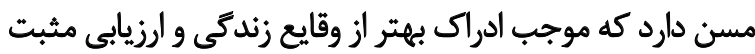

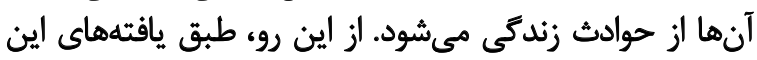

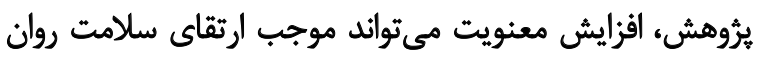

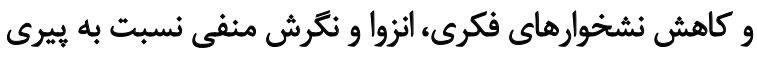

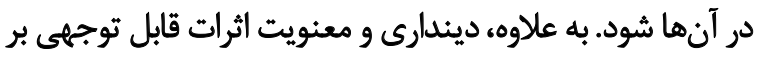

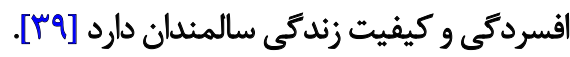

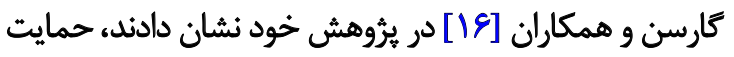

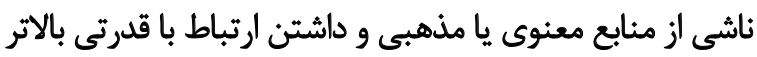

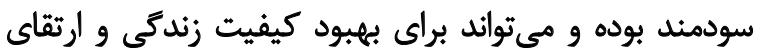

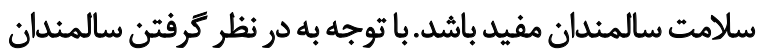

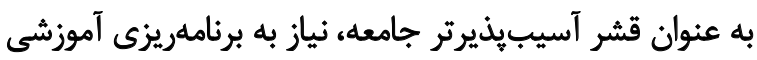

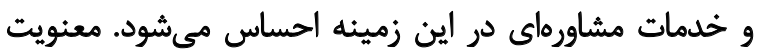

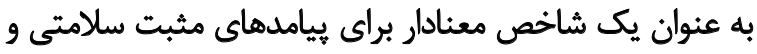

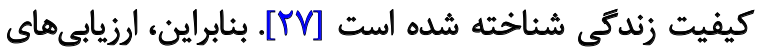

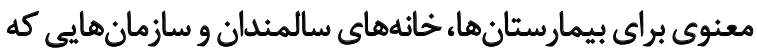

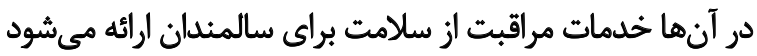

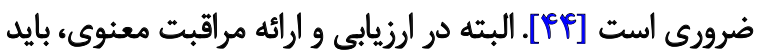

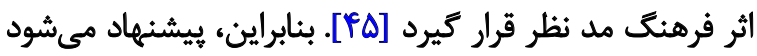

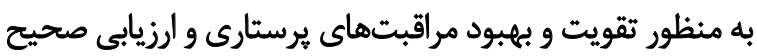

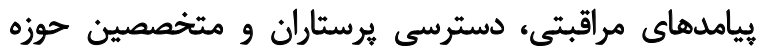

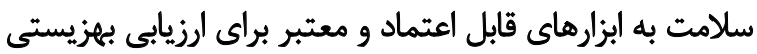

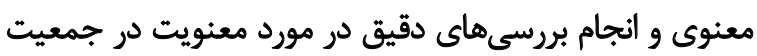
سالمندى مورد توجه جدى قريى قرار تيرد.

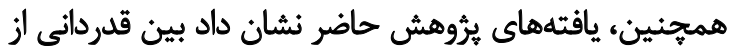

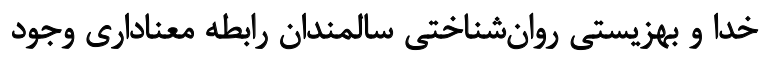

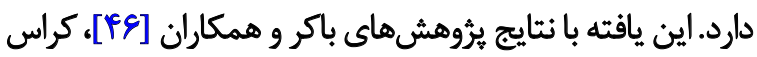

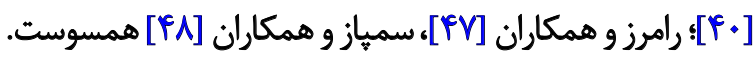

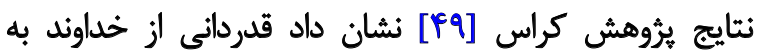

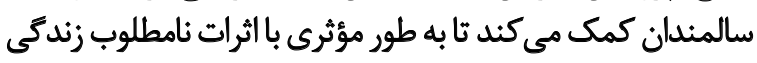

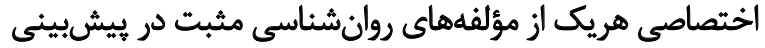

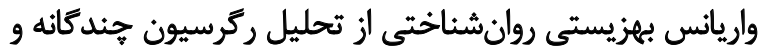

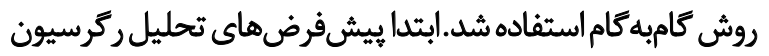

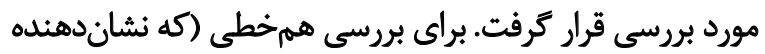

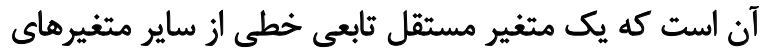

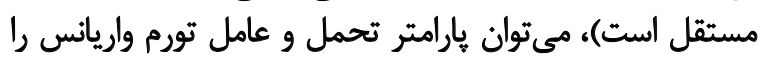

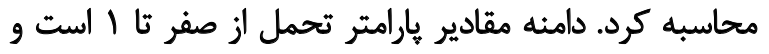

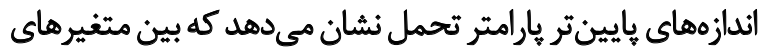

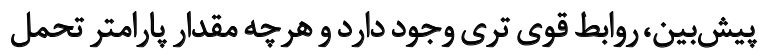

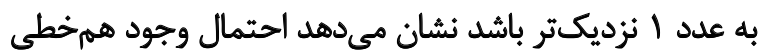

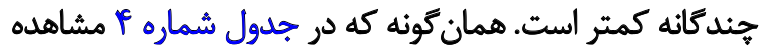

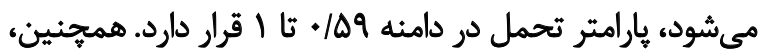

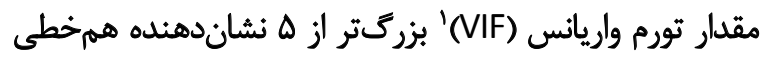

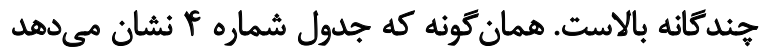

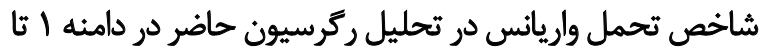

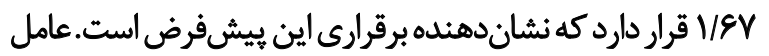

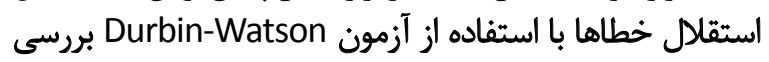

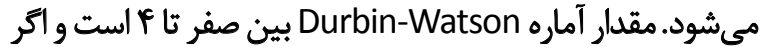

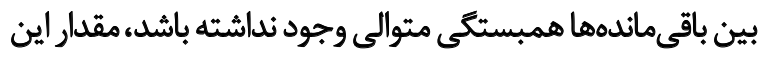

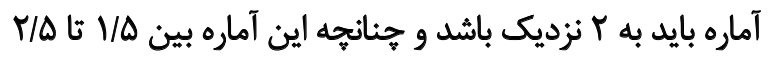

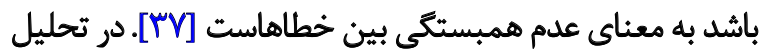

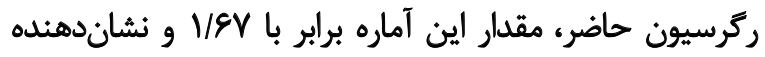

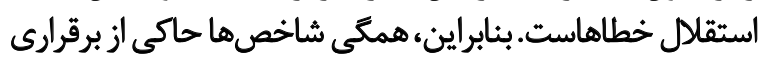

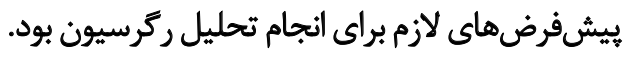
نتايج تحليل ركرسيون كامبه كام نشان داد كه به به ترتيب،

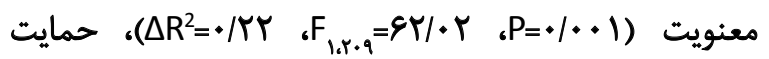

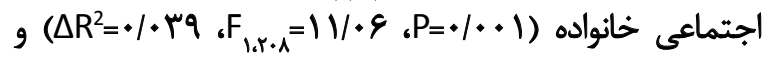

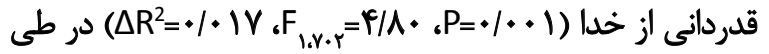

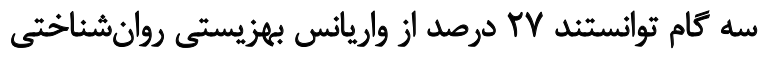

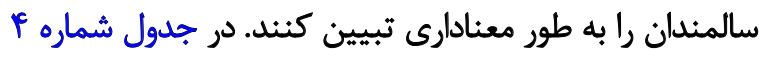

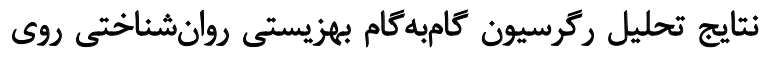

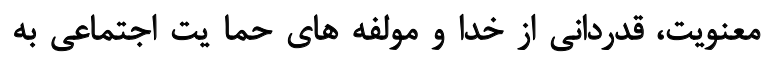
كزارش شده است. ضرايب استاندارد تحليل ركرسيون در جدول شماره f من نشان

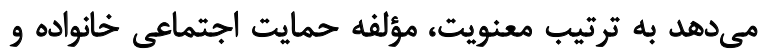

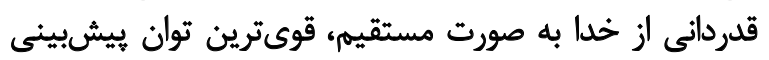
بهزيستى روانشناختى در سالمندان رادارند.

\&)

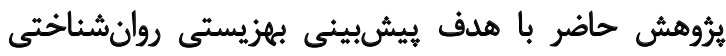

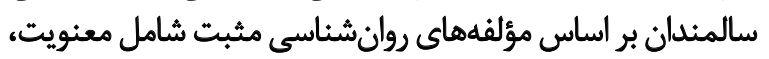

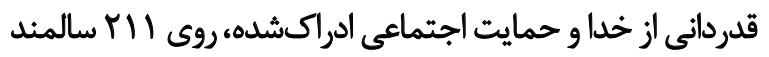

1. Variance Inflation Factor 
ادراكشده بهويره از جانب خانواده با بهزيستى روانشناختى

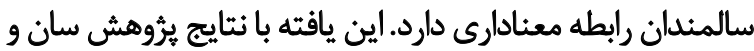

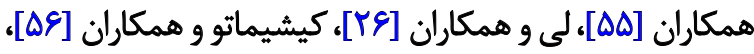

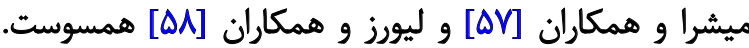

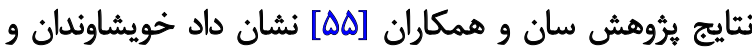

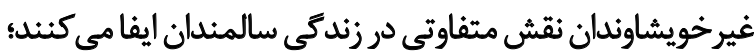

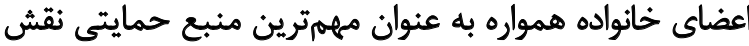

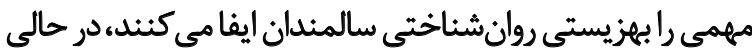

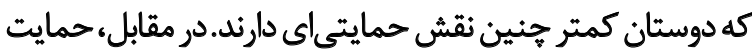

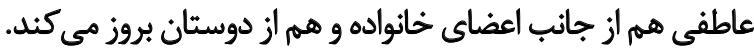

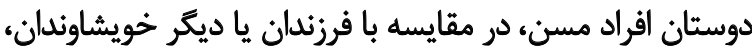

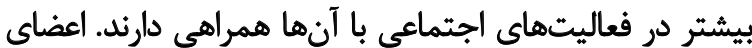

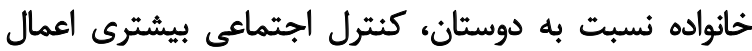

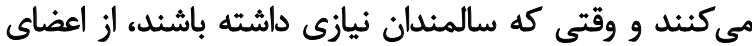

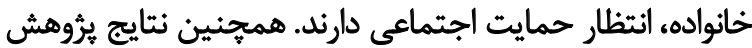

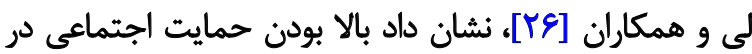

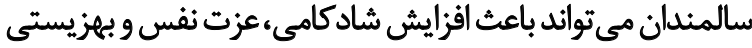
روانشناختى در اين افراد شود.

ميزان حمايتهايى كه سالمندان از خانواده و دوستان خود

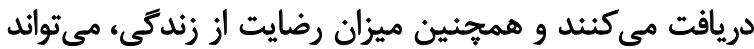

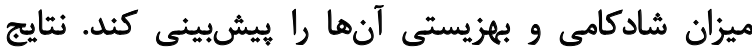

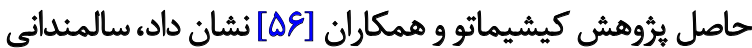

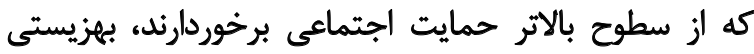

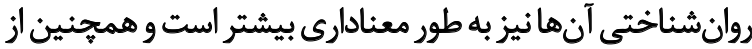

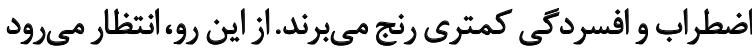

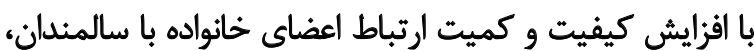

بتوان حمايت مورد نياز آنان را ازئ طريق خانوان إده تأمين كرد.

شواهد يُوهشى موجود حاكى از آن است كه اقدامات مراقبتى

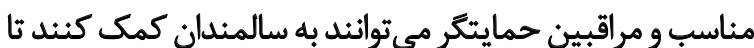

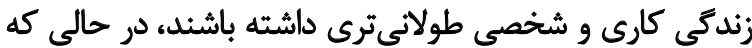

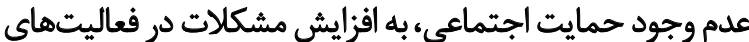

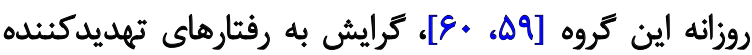

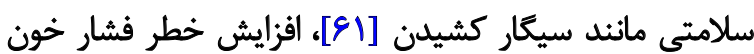

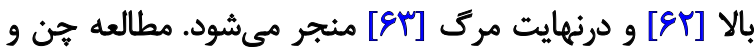

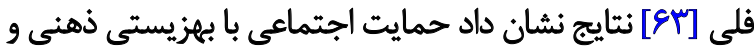

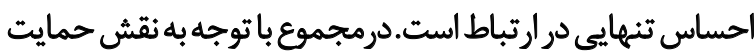

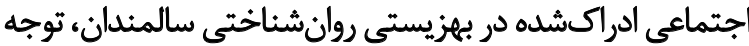

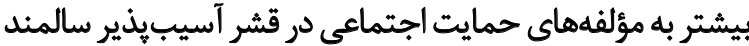
احساس مىشود و حمايت اجتماعى مى توائد به بهائ بهبود شرايط روانى اجتماعى سالمندان كمك كند.

$$
\text { نتيجلكيرى نمهايى }
$$

نتيجه كلى كه از اين تحقيق مى توان به دست آورد اين است كه

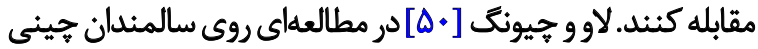
در كروه سنى

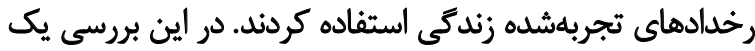

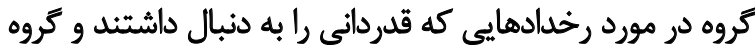

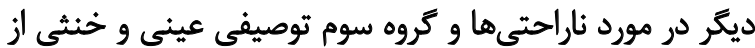

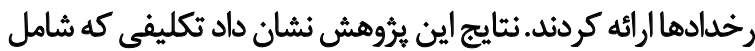

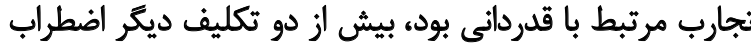

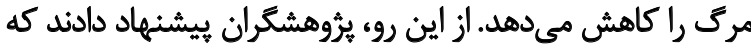

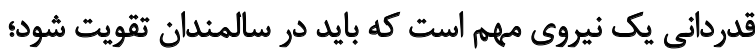

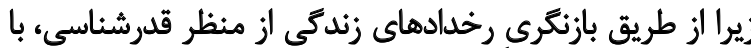

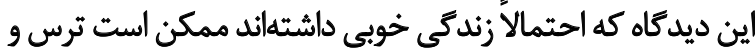

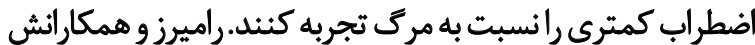

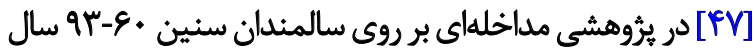

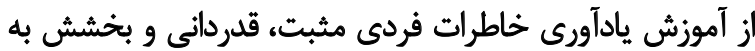

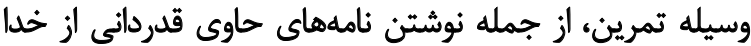

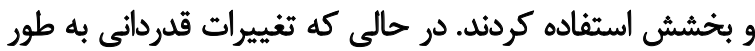

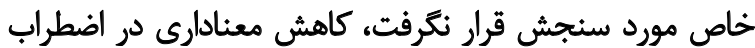

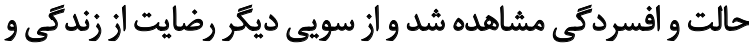

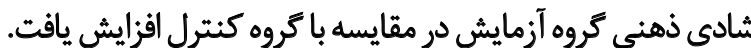

يك بررسى با هدف ارزيابى سالمندى موفق از طريق

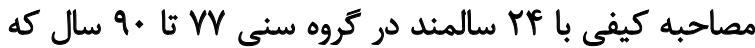

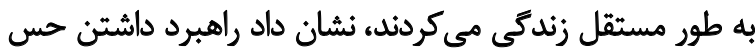

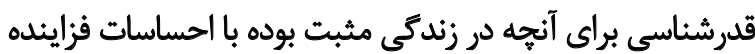

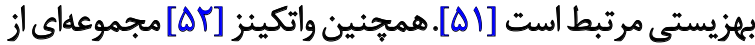

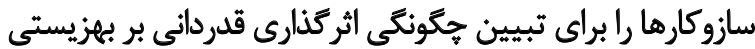

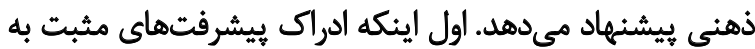

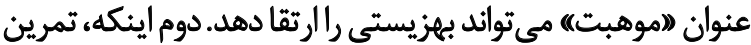

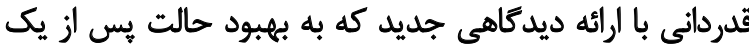

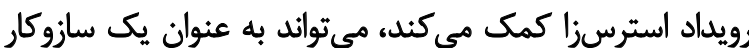

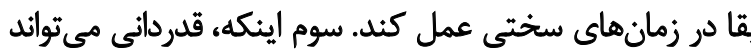
دسترسيذيرى خاطرات خوشايند را افزايش دهد سند. يرؤهشكران متعددى بروجود ارثباط ميان بخشش، قدرشناسى،

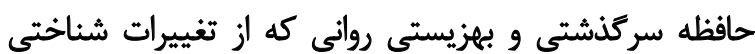

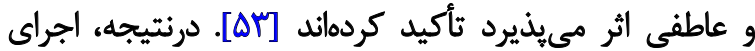

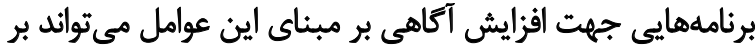

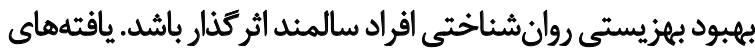

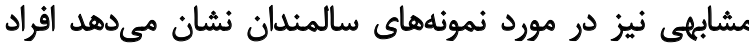

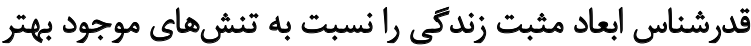

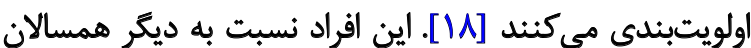

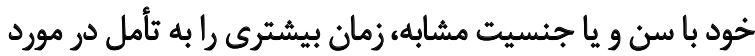

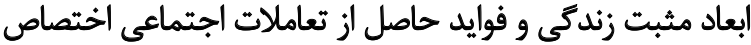

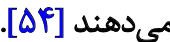
به علاوه، نتايج يُوهش حاضر نشان داد حمايت اجتماعى 


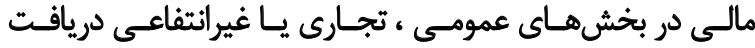

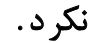

\section{مشاركت نويسند مكان}

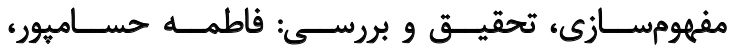

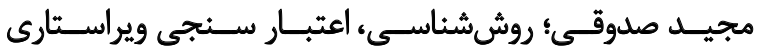

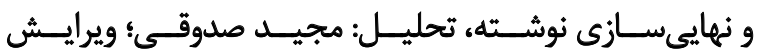

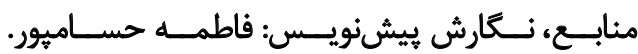

$$
\text { تعارض منافع }
$$

بنابر اظهار نويسندكان، اين مقاله تعارض منافع ندارد.
معنويت وقدردانى از خدا مي توانند به عنوان شاخص معنادار براى

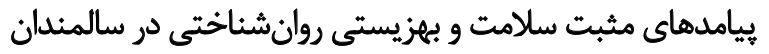

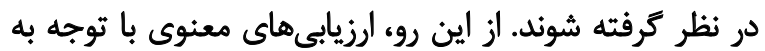

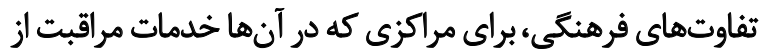

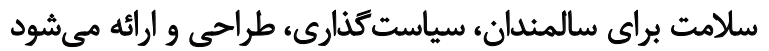

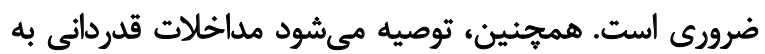

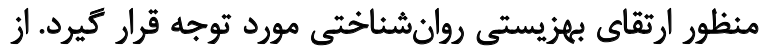

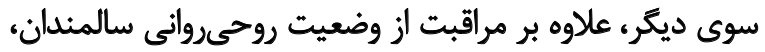

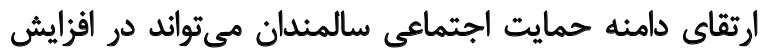

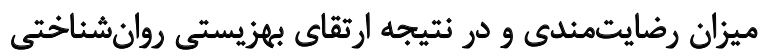

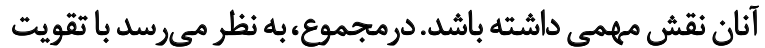

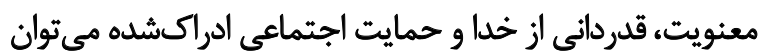

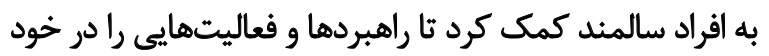

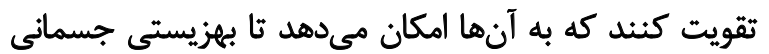

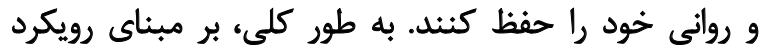

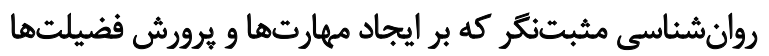

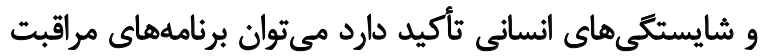
بهداشتى سالمندان را به سوى ييشكيرى و مقابله با مشكلات

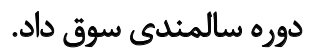

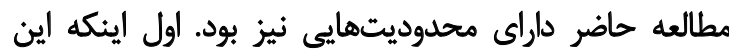

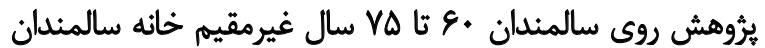

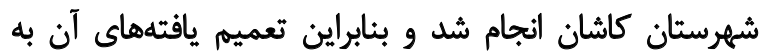

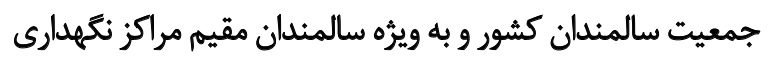

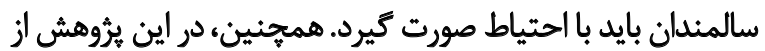

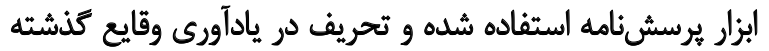

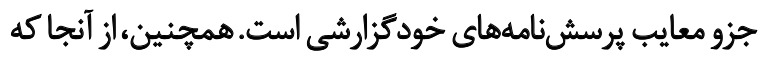

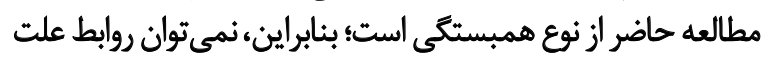

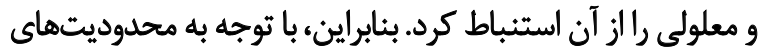

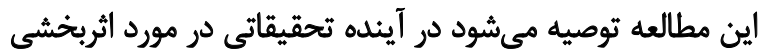

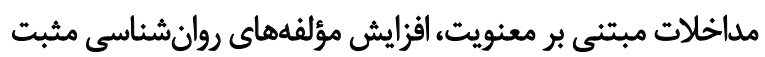

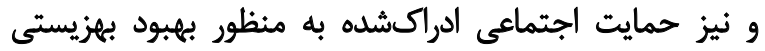
روانشناختى سالمندان انجام و مورد بررسى قرار كيرد.

مالاحظات اخلاقي

\section{ييروى أز اصول اخلاق بؤوهش}

كليه اصول اخلاق ثرؤهش در اين تحقيق رعايت شده است.

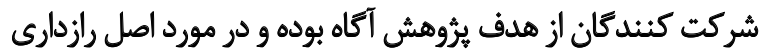

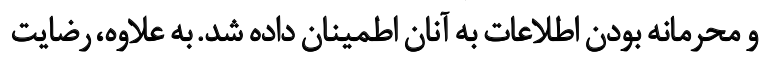

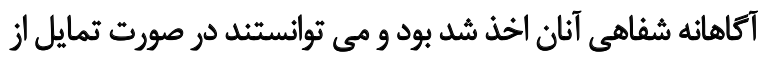
شركت در يُوهش امتناع كنند.

$$
\text { حامى مالى }
$$

ايسن تحقيـق هيسج كونسه كمـك مالـى از سـازمانهاى تأميـن 


\section{References}

[1] Westerhof GJ, Bohlmeijer ET, van Beljouw IMJ, Pot AM. Improvement in personal meaning mediates the effects of a life review intervention on depressive symptoms in a randomized controlled trial. The Gerontologist. 2010; 50(4):541-9. [DOI:10.1093/ geront/gnp168] [PMID]

[2] Baird BM, Lucas RE, Donnellan MB. Life satisfaction across the lifespan: Findings from two nationally representative panel studies. Social Indicators Research. 2010; 99(2):183-203. [DOI:10.1007/ s11205-010-9584-9] [PMID] [PMCID]

[3] Steptoe A, Deaton A, Stone AA. Subjective wellbeing, health, and ageing. Lancet. 2015; 385(9968):640-8. [DOI:10.1016/S01406736(13)61489-0]

[4] Shtompel N, Whiteman K, Ruggiano N. Negative feelings and help seeking among older adults with chronic conditions. Journal of Gerontological Social Work. 2014; 57(8):810-24. [DOI:10.1080/0 1634372.2014.898008] [PMID]

[5] Corcoran J, Brown E, Davis M, Pineda M, Kadolph J, Bell H. Depression in older adults: A meta-synthesis. Journal of Gerontological Social Work. 2013; 56(6):509-34. [DOI:10.1080/01634372 .2013.811144] [PMID]

[6] Park N, Peterson C, Seligman MEP. Strengths of character and well-being. Journal of Social and Clinical Psychology. 2004; 23(5):603-19. [DOI:10.1521/jscp.23.5.628.50749]

[7] Kim ES, Park N, Peterson CH. Dispositional optimism protects older adults from stroke: The health and retirement study. Stroke. 2011; 42(10):2855-9. [DOI:10.1161/STROKEAHA.111.613448] [PMID]

[8] Ferring D, Balducci C, Burholt V, Wenger C, Thissen F, Weber $G$, et al. Life satisfaction of older people in six European countries: Findings from the European study on adult well-being. European Journal of Ageing. 2004; 1(1):15-25. [DOI:10.1007/s10433-0040011-4] [PMID] [PMCID]

[9] Killen A, Macaskill A. Using a gratitude intervention to enhance well-being in older adults. Journal of Happiness Studies. 2015; 16(4):947-64. [DOI:10.1007/s10902-014-9542-3]

[10] Godoy-Izquierdo D, Moreno RL, Vázquez Pérez ML, Serrano FA, Godoy García JF. Correlates of happiness among older Spanish institutionalised and non-institutionalised adults. Journal of Happiness Studies. 2013; 14(2):389-414. [DOI:10.1007/s10902-012-9335-5]

[11] Izquierdo DG, Martínez A, Godoy JF. Balance afectivo en hombres y mujeres: Implicaciones de la edad y el sexo. Behavioral Psychology/Psicología Conductual. 2009; 17(2):299-319. https://www.behavioralpsycho.com/wp-content/uploads/2020/04/07.Godoy_17-2r.pdf

[12] Windsor TD, Burns RA, Byles JE. Age, physical functioning, and affect in midlife and older adulthood. Journals of Gerontology Series B: Psychological Sciences and Social Sciences. 2013; 68(3):395-9. [DOI:10.1093/geronb/gbs088] [PMID]

[13] Oman D. Spiritual practice, health promotion, and the elusive soul: Perspectives from public health. Pastoral Psychology. 2011; 60(6):897-906. [DOI:10.1007/s11089-011-0359-2]

[14] Daaleman TP, Frey BB. The spirituality index of well-being: A new instrument for health-related quality-of-life research. The Annals of Family Medicine. 2004; 2(5):499-503. [DOI:10.1370/ afm.89] [PMID] [PMCID]
[15] Erichsen NB, Büssing A. Spiritual needs of elderly living in residential/nursing homes. Evidence-Based Complementary and Alternative Medicine. 2013; 2013:913247. [DOI:10.1155/2013/913247] [PMID] [PMCID]

[16] Garssen B, Visser A, de Jager Meezenbroek E. Examining whether spirituality predicts subjective well-being: How to avoid tautology. Psychology of Religion and Spirituality. 2016; 8(2):141-8. [DOI:10.1037/rel0000025]

[17] Emmons RA, Mishra A. Why gratitude enhances well-being What we know, what we need to know. In: Sheldon MK, Kashdan TB, Steger MF, editors. Designing Positive Psychology: Taking Stock and Moving Forward. Oxford: Oxford University Press; 2011. [DOI:10.1093/acprof:oso/9780195373585.003.0016]

[18] Emmons RA, McCullough ME. Counting blessings versus burdens: An experimental investigation of gratitude and subjective well-being in daily life. Journal of Personality and Social Psychology. 2003; 84(2):377-89. [DOI:10.1037/0022-3514.84.2.377] [PMID]

[19] Emmons RA, Crumpler CA. Gratitude as a human strength: Appraising the evidence. Journal of Social and Clinical Psychology. 2000; 19(1):56-69. [DOI:10.1521/jscp.2000.19.1.56]

[20] Levin J. Is depressed affect a function of one's relationship with god?: Findings from a study of primary care patients. The International Journal of Psychiatry in Medicine. 2002; 32(4):379-93. [DOI:10.2190/3183-WPYV-3KYY-K3V7] [PMID]

[21] Pargament KI, Koenig HG, Tarakeshwar N, Hahn J. Religious struggle as a predictor of mortality among medically ill elderly patients: A 2-year longitudinal study. Archives of Internal Medicine. 2001; 161(15):1881-5. [DOI:10.1001/archinte.161.15.1881] [PMID]

[22] McAdams DP, Bauer JJ. Gratitude in modern life: Its manifestations and development. In: Emmons RA, McCullough ME, rditors. Series in affective science. Oxford: Oxford University Press; 2004. [DOI:10.1093/acprof:o so/9780195150100.003.0005]

[23] Carstensen LL, Isaacowitz DM, Charles ST. Taking time seriously: A theory of socioemotional selectivity. The American Psychologist. 1999; 54(3):165-81. [DOI:10.1037/0003-066X.54.3.165] [PMID]

[24] Kashdan TB, Mishra A, Breen WE, Froh JJ. Gender differences in gratitude: Examining appraisals, narratives, the willingness to express emotions, and changes in psychological needs. Journal of Personality. 2009; 77(3):691-730. [DOI:10.1111/j.14676494.2009.00562.x] [PMID]

[25] Mazlumi SS, Saeedi M, Vahedian M, Jalalpour Z, Kiani MA [The effect burnout on social support and self-esteem in health care workers in Yazd city (Persian)]. Occupational Medicine

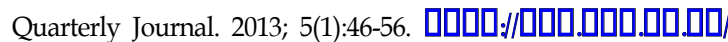

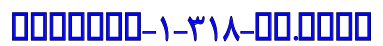

[26] Lee JE, Kahana B, Kahana E. Social support and cognitive functioning as resources for elderly persons with chronic arthritis pain. Aging \& Mental Health. 2016; 20(4):370-9. [DOI:10.1080/136 07863.2015.1013920] [PMID] [PMCID]

[27] Bai M, Lazenby M. A systematic review of associations between spiritual well-being and quality of life at the scale and factor levels in studies among patients with cancer. Journal of Palliative Medicine. 2015; 18(3):286-98. [DOI:10.1089/jpm.2014.0189] [PMID] [PMCID] 
[28] Lena MML, Rivera-Ledesma A. Variables con alto valor adaptativo en el desajuste psicológico del adulto mayor. Journal of Behavior, Health \& Social Issues. 2009; 1(1):59-67. [DOI:10.22201/ fesi.20070780.2009.1.1.382]

[29] Phillips WJ, Ferguson SJ. Self-compassion: A resource for positive aging. Journals of Gerontology Series B: Psychological Sciences and Social Sciences. 2012; 68(4):529-39. [DOI:10.1093/geronb/ gbs091] [PMID]

[30] Jopp D, Smith J. Resources and life-management strategies as determinants of successful aging: On the protective effect of selection, optimization, and compensation. Psychology and Aging. 2006; 21(2):253-65. [DOI:10.1037/0882-7974.21.2.253] [PMID]

[31] Roh S, Lee KH, Yoon DP. General well-being of korean immigrant elders: The significance of religiousness/spirituality and social support. Journal of Social Service Research. 2013; 39(4):483-97. [DOI:10.1080/01488376.2012.709451]

[32] Paloutzian RF, Ellison CW. Loneliness, spiritual well-being and quality of life. In: Peplau LA, Perlman D, editors. Loneliness: A Sourcebook of Current Theory, Research and Therapy. New York: John Wiley \& Sons; 1982. [DOI:10.1037/t00534-000] [PMID]

[33] Zimet GD, Dahlem NW, Zimet SG, Farley GK. The multidimensional scale of perceived social support. Journal of Personality Assessment. 1988; 52(1):30-41. [DOI:10.1207/s15327752jpa5201_2]

[34] Ryff CD. Happiness is everything, or is it? Explorations on the meaning of psychological well-being. Journal of Personality and Social Psychology. 1989; 57(6):1069-81. [DOI:10.1037/00223514.57.6.1069]

[35] Allahbakhshian M, Jaffarpour M, Parvizy S, Haghani H. [A survey on relationship between spiritual wellbeing and quality of life in multiple sclerosis patients (Persian)]. Zahedan Journal of Research in Medical Sciences. 2010; 12(3):29-33. https:/ / sites. kowsarpub.com/zjrms/articles/94299.html

[36] Bruwer B, Emsley R, Kidd M, Lochner C, Seedat S. Psychometric properties of the multidimensional scale of perceived socia support in youth. Comprehensive Psychiatry. 2008; 49(2):195-201. [DOI:10.1016/j.comppsych.2007.09.002] [PMID]

[37] Behnamfar R, Rasti A. [Linear regression, the normal distribution of error values or normal distribution of the dependent variable? (Persian)]. Iranian Journal of Medical Education. 2015 15:263-5. http:/ /ijme.mui.ac.ir/article-1-3780-fa.html

[38] Wilt JA, Grubbs JB, Exline JJ, Pargament KI. Personality, religious and spiritual struggles, and well-being. Psychology of Religion and Spirituality. 2016; 8(4):341-51. [DOI:10.1037/rel0000054]

[39] Moon YS, Kim DH. Association between religiosity/spirituality and quality of life or depression among living-alone elderly in a South Korean city. Asia-Pacific Psychiatry. 2013; 5(4):293-300 [DOI:10.1111/appy.12025] [PMID]

[40] Krause N, Pargament KI, Hill PC, Ironson G. Humility, stressful life events, and psychological well-being: Findings from the landmark spirituality and health survey. The Journal of Positive Psychology. 2016; 11(5):499-510. [DOI:10.1080/17439760.2015.112 7991]

[41] Cowlishaw S, Niele S, Teshuva K, Browning C, Kendig H. Older adults' spirituality and life satisfaction: A longitudinal test of social support and sense of coherence as mediating mechanisms. Ageing \& Society. 2013; 33(7):1243-62. [DOI:10.1017/ S0144686X12000633]
[42] Agli O, Bailly N, Ferrand C. Spirituality and religion in older adults with dementia: A systematic review. International Psychogeriatrics. 2015; 27(5):715-25. [DOI:10.1017/S1041610214001665] [PMID]

[43] Wu LF, Koo M. Randomized controlled trial of a six-week spiritual reminiscence intervention on hope, life satisfaction, and spiritual well-being in elderly with mild and moderate dementia. International Journal of Geriatric Psychiatry. 2016; 31(2):120-7 [DOI:10.1002/gps.4300] [PMID]

[44] Hodge DR, Horvath VE, Larkin H, Curl AL. Older adults' spiritual needs in health care settings: A qualitative meta-synthesis. Research on Aging. 2012; 34(2):131-55. [DOI:10.1177/0164027511411308]

[45] Cockell N, McSherry W. Spiritual care in nursing: An overview of published international research. Journal of Nursing Management. 2012; 20(8):958-69. [DOI:10.1111/j.1365-2834.2012.01450.x] [PMID]

[46] Booker JA, Dunsmore JC. Profiles of wisdom among emerging adults: Associations with empathy, gratitude, and forgiveness. The Journal of Positive Psychology. 2016; 11(3):315-25. [DOI:10.10 80/17439760.2015.1081970

[47] Ramírez E, Ortega AR, Chamorro A, Colmenero JM. A program of positive intervention in the elderly: Memories, gratitude and forgiveness. Aging \& Mental Health. 2014; 18(4):463-70. [DOI :10.1080/13607863.2013.856858] [PMID]

[48] Sapmaz F, Yıldırım M, Topcuoğlu P, Nalbant D, Sızır U. Gratitude, forgiveness and humility as predictors of subjective well-being among university students. International Online Journal of Educational Sciences. 2016; 8(1):38-47. [DOI:10.15345/ iojes.2016.01.004

[49] Krause N. Gratitude toward God, stress, and health in late life. Research on Aging. 2006; 28(2):163-83. [DOI:10.1177/0164027505284048]

[50] Lau RWL, Cheng ST. Gratitude lessens death anxiety. European Journal of Ageing. 2011; 8(3):169-75. [DOI:10.1007/s10433011-0195-3] [PMID] [PMCID]

[51] Hörder HM, Frändin K, Larsson MEH. Self-respect through ability to keep fear of frailty at a distance: Successful ageing from the perspective of community-dwelling older people. International Journal of Qualitative Studies on Health and Well-Being. 2013; 8:20194. [DOI:10.3402/qhw.v8i0.20194] [PMID] [PMCID]

[52] Watkins PC, Grimm DL, Kolts R. Counting your blessings: Positive memories among grateful persons. Current Psychology. 2004; 23(1):52-67. [DOI:10.1007/s12144-004-1008-z]

[53] Bono G, McCullough ME. Positive responses to benefit and harm: Bringing forgiveness and gratitude into cognitive psychotherapy. Journal of Cognitive Psychotherapy. 2006; 20(2):147-58. [DOI:10.1891/jcop.20.2.147]

[54] König S, Glück J. "Gratitude is with me all the time": How gratitude relates to wisdom. Journals of Gerontology Series B: Psychological Sciences and Social Sciences. 2014; 69(5):655-66. [DOI:10.1093/geronb/gbt123] [PMID] [PMCID]

[55] Sun Y, Zhang D, Yang Y, Wu M, Xie H, Zhang J, et al. Social support moderates the effects of self-esteem and depression on quality of life among Chinese rural elderly in nursing homes. Archives of Psychiatric Nursing. 2017;31(2):197-204. [DOI:10.1016/j. apnu.2016.09.015] [PMID] 
[56] Kishimoto Y, Okamoto N, Saeki K, Tomioka K, Obayashi K, Komatsu M, et al. Bodily pain, social support, depression symptoms and stroke history are independently associated with sleep disturbance among the elderly: A cross-sectional analysis of the Fujiwara-kyo study. Environmental Health and Preventive Medicine. 2016; 21(5):295-303. [DOI:10.1007/s12199-016-0529-z] [PMID] [PMCID]

[57] Mishra S, Pandey D, Khan KAZ, Joby PA, Jha M. Predicting effect of social support on psychological wellbeing in elderly. Indian Journal of Health \& Wellbeing. 2014; 5(10):1188-90. https:/ / www.academia.edu/23849131/Predicting_effect_of_social_support_on_psychological_wellbeing_in_elderly

[58] Lieverse R, de Vries R, Hoogendoorn AW, Smit JH, Hoogendijk WJG. Social support and social rhythm regularity in elderly patients with major depressive disorder. The American Journal of Geriatric Psychiatry. 2013; 21(11):1144-53. [DOI:10.1016/j. jagp.2013.01.052] [PMID]

[59] Buchman AS, Boyle PA, Wilson RS, James BD, Leurgans SE, Arnold SE, et al. Loneliness and the rate of motor decline in old age: The rush memory and aging project, a community-based cohort study. BMC Geriatrics. 2010; 10:77. [DOI:10.1186/1471-231810-77] [PMID] [PMCID]

[60] Perissinotto CM, Cenzer IS, Covinsky KE. Loneliness in older persons: A predictor of functional decline and death. Archives of Internal Medicine. 2012; 172(14):1078-83. [DOI:10.1001/archinternmed.2012.1993] [PMID] [PMCID]

[61] Shankar A, McMunn A, Banks J, Steptoe A. Loneliness, social isolation, and behavioral and biological health indicators in older adults. Health Psychology. 2011; 30(4):377-85. [DOI:10.1037/ a0022826] [PMID]

[62] Hawkley LC, Thisted RA, Masi CM, Cacioppo JT. Loneliness predicts increased blood pressure: 5-year cross-lagged analyses in middle-aged and older adults. Psychology and Aging. 2010; 25(1):132-41. [DOI:10.1037/a0017805] [PMID] [PMCID]

[63] Chen Y, Feeley TH. Social support, social strain, loneliness, and well-being among older adults. Journal of Social and Personal Relationships. 2014; 31(2):141-61. [DOI:10.1177/0265407513488728] 
This Page Intentionally Left Blank 